\title{
Identification of differentially expressed non-coding RNAs and mRNAs involved in Qi stagnation and blood stasis syndrome
}

\author{
GUANG CHEN $^{1,2^{*}}$, JIALIANG GAO ${ }^{2 *}$, HAOQIANG HE ${ }^{1,2}, \mathrm{CHAO} \mathrm{LIU}^{1,2}$, \\ YONGMEI LIU ${ }^{2}$, JUN LI ${ }^{2}$ and JIE WANG ${ }^{2}$ \\ ${ }^{1}$ Graduate School, Beijing University of Chinese Medicine, Beijing 100029; ${ }^{2}$ Department of Cardiology, \\ Guang'anmen Hospital, China Academy of Chinese Medical Sciences, Beijing 100053, P.R. China
}

Received January 23, 2018; Accepted September 13, 2018

DOI: $10.3892 /$ etm.2018.7068

\begin{abstract}
Qi stagnation and blood stasis syndrome (QSBSS) is a common Zheng in Traditional Chinese Medicine (TCM), describes the condition of unsmooth flow of Qi and blood, which manifests as distending pain in a fixed body part and emotional disorders, including irritability and depression. However, the underlying molecular mechanisms remain largely elusive. RNAs are the connection between DNA and proteins, which reflect the interaction between the genotypes and the phenotype. Of note, non-coding (nc)RNA is a type of RNA that is not translated into any protein, but has regulatory functions. Despite the growing interest in exploring the biological basis of TCM Zhengs, the specific roles of ncRNAs in QSBSS have remained largely elusive. In the present study, next-generation sequencing was performed to investigate the ncRNA profile in patients with three different types of disease, but who had QSBSS. A total of 104 long non-coding RNAs, 2 circular RNAs and 697 mRNAs were identified to be significantly differentially expressed in QSBSS patients. Further bioinformatics analysis revealed that the most significantly enriched pathways by the differentially expressed RNAs in QSBSS were the sphingolipid signaling pathway, the neurotrophin signaling pathway, 5'AMP-activated protein kinase and endocytosis. In addition, a network pharmacology analysis indicated that several of the differentially expressed RNAs were included in the targets of TCM herbs for treating QSBSS. The present study was the first to identify ncRNAs that are deregulated in QSBSS by next-generation
\end{abstract}

Correspondence to: Professor Jie Wang, Department of Cardiology, Guang'anmen Hospital, China Academy of Chinese Medical Sciences, 5 Beixian Ge, Xi Cheng, Beijing 100053, P.R. China

E-mail: wangjie0103@126.com

*Contributed equally

Key words: Qi stagnation and blood stasis syndrome, non-coding RNAs, sequencing, expression profiles, Traditional Chinese Medicine sequencing technology. The results may offer insight into the biological basis of TCM Zheng and the optimization of ancient formulae, as well as the discovery of novel drugs, to pave the way toward advanced TCM theory and improved health care delivery.

\section{Introduction}

It is a common phenomenon that the symptoms and signs are slightly different among patients with the same disease (1). For instance, coronary heart disease (CHD) manifests as chest pain (2). However, certain patients suffer from a stabbing pain, while others have complaints of a burning pain (3). Within the framework of Traditional Chinese Medicine (TCM) theory, practitioners always prescribe a medical formula based on symptoms and signs (4). In fact, TCM practitioners may divide patients with the same disease into several subtypes according to these slight differences and prescribe the corresponding formulae. In TCM theory, these subtypes are known as Zheng (5). It is renowned that the advancement of Zheng depends on the genes inherited from the parents, the lifestyle and the environmental conditions, which may differ between individual patients, and therefore, different types of Zheng exist (6). The first rule of Zheng is that one type of disease may be divided into several different Zheng (7).

A disequilibrium of the body's original condition is accountable for the development of Zheng, and certain deviations from the equilibrium may lead to certain types of disease and manifestations thereof (8). For instance, Qi stagnation and blood stasis syndrome (QSBSS), a type of Zheng, describes the condition of unsmooth flow of Qi and blood, which may occur in diseases including CHD, chronic gastritis (CG) and rheumatoid arthritis (RA) (9). This exemplifies the second rule of Zheng, namely that one type of Zheng may be the basis of several different types of disease (7). However, the western scientific basis for this theory, including the substantial essence of this Zheng, remains to be fully elucidated. Previous studies in the field mainly focus on the subtypes of one disease based on Zheng, while those on the biological basis of the same Zheng in different diseases are scarce $(10,11)$.

The genetic profile of an individual provides information on what could theoretically happen in the body, while protein expression profiles are representative of the processes that are 
active in the body at the time-point of determination (12). Of note, RNAs are the connection between DNAs and proteins, which reflect the interaction of the genotype and the environment (8). Among the different types of RNAs, messenger RNAs (mRNAs) are transcripts of DNA and the information they carry is transferred by their translation into proteins in accordance with the central dogma (13). Long non-coding RNAs (lncRNAs) are a class of non-protein-coding RNAs of $>200$ nucleotides in length, and they are known to have roles in controlling chromatin structure, transcriptional regulation and post-transcriptional processing $(14,15)$. Furthermore, circular RNAs (circRNAs), which have drawn an increasing amount of attention recently, are a type of non-canonical form of alternative splicing and are more stable than linear RNAs $(16,17)$. Network pharmacology studies based on the theory of systems biology may be used for the preliminary validation of the interaction between TCM herbs and RNA targets (18). Due to its core methods based on the holistic view and the dynamic balance of the human body, network pharmacology is useful for the study of TCM herbs with complex mechanisms of action (19). In the present study, a network pharmacology analysis was employed to preliminarily validate the differentially expressed (DE) RNAs in subjects with QSBSS. The purpose of the present study was to explore the biological basis of one common Zheng, QSBSS, from the perspective of ncRNAs and mRNAs in a diversity of associated diseases.

\section{Materials and methods}

Patients and samples. The protocol of the present study was approved by the Ethics Committee of Guang'anmen Hospital (Beijing, China). The study included 5 patients with CHD and QSBSS (group a), 5 patients with CG and QSBSS (group b), 5 patients with RA and QSBSS (group c) and 5 completely healthy subjects (control) who presented at Guang'anmen Hospital affiliated to the China Academy of Chinese Medical Sciences (Beijing, China) between October 2016 and December 2016. Informed consent was provided by all of the participants prior to the study.

The diagnosis of QSBSS was based on the judgement of an expert clinician according to the standard criteria approved by the China Food and Drug Administration (20) and the scaled diagnosis criteria, which is used to diagnose Qi Stagnation and Blood Stasis Syndrome (21). The criteria for QSBSS included primary symptoms and signs [i) distending pain; ii) tingling sensation anywhere on the body; iii) pain in a fixed place; iv) pain aggravated by touch; v) lumps in the body; vi) self-reported irritability; vii) clinical depression diagnosed by a psychiatrist], secondary symptoms and signs [i) loss of appetite; ii) paleness of the face; iii) hemorrhagic spots on any part of the body], TCM tongue presentations [i) purple, dusky tongue; ii) stasis macules or stasis spots on the tongue] and pulse presentation [i) choppy pulse; ii) wiry pulse] (22). A patient was diagnosed with QSBSS if they had $>2$ of the primary symptoms and signs, $>1$ of the secondary symptoms and signs, and 1 of the tongue or pulse presentations. The diagnosis of CHD, RA and CG were based on guidelines for the diagnosis and management of patients with stable ischaemic heart disease (23), the diagnosis and treatment for rheumatoid arthritis (24) and the consensus of chronic gastritis (25), respectively. From each of the patients, blood samples (3-4 ml) were collected in EDTA-containing tubes.

RNA isolation and library preparation. RNA degradation and contamination was monitored on $1 \%$ agarose gels. RNA purity was confirmed using the Agencourt AMPure XP (cat. no. A63881; Beckman Coulter, Brea, CA, USA). RNA integrity was assessed using the RNA Nano 6000 Assay Kit for the Bioanalyzer 2100 system (cat. no. 5067-1511; Agilent Technologies, Inc., Santa Clara, CA, USA). The methods of IncRNA quantification were identical to the conventional methods for mRNAs. Quantification of circRNAs was performed with exonuclease to exclude non-circRNAs. After RNA extraction, the total RNA was digested with TruSeq Stranded Total RNA with Ribo-Zero Gold (cat. no. 15021048; Illumina, Inc., San Diego, CA, USA). The sample was then subjected to reverse transcription using SuperScript II Reverse Transcriptase (cat. no. 18064014; Invitrogen; Thermo Fisher Scientific, Inc., Waltham, MA, USA).

A total of $3 \mu \mathrm{g}$ RNA per sample was used as input material for the RNA sample preparations. First, ribosomal (r)RNA was removed using Agencourt RNAClean XP (cat. no. A63987; Beckman Coulter), and rRNA-free material was cleaned up by ethanol precipitation. Subsequently, sequencing libraries were generated using the rRNA-depleted RNA. Library preparation was performed according to manufacturer's protocol (Illumina). In brief, fragmentation was performed using divalent cations at $94^{\circ} \mathrm{C}$ in $5 \mu \mathrm{lRNA}$ Binding Buffer and $5 \mu \mathrm{l}$ rRNA Removal Mix-Gold. First-strand complementary (c)DNA was synthesized using $8 \mu \mathrm{l}$ First Strand Synthesis Act D Mix and SuperScript II Reverse Transcriptase. Second-strand cDNA synthesis was performed using $5 \mu \mathrm{l}$ End Repair Control ( $2 \mu 1$ End Repair Control $+98 \mu 1$ Resuspension Buffer). The reaction buffer contained deoxynucleoside triphosphate where thymine was replaced by uracil. After adenylation of the 3 'ends of the DNA fragments, an adaptor with a hairpin loop structure was ligated to prepare for hybridization. To select cDNA fragments of preferentially $150-200 \mathrm{bp}$ in length, the library fragments were purified using the AMPure XP system (cat. no. A63881; Beckman Coulter). The DNA fragment was enriched by using $5 \mu$ l polymerase chain reaction (PCR) Primer Cocktail and $25 \mu \mathrm{l}$ PCR Master Mix prior to performing PCR. The mixture was incubated in the following conditions: $98^{\circ} \mathrm{C}$ for $30 \mathrm{sec}, 15$ cycles $98^{\circ} \mathrm{C}$ for $10 \mathrm{sec}, 60^{\circ} \mathrm{C}$ for $30 \mathrm{sec}, 72^{\circ} \mathrm{C}$ for $30 \mathrm{sec}$, then $72^{\circ} \mathrm{C}$ for $5 \mathrm{~min}$ and held at $10^{\circ} \mathrm{C}$. Finally, products were purified (AMPure XP system) and library quality was assessed on the Agilent Bioanalyzer 2100 system.

Sequencing of RNAs and DE RNAs analysis. The clustering of the index-coded samples was performed on a cBot Cluster Generation System using TruSeq PE Cluster Kit v3cBot-HS (Illumina) according to the manufacturer's protocols. After cluster generation, the libraries were sequenced on an Illumina HiSeq 2,500 platform, and 125 bp paired-end and $50 \mathrm{bp}$ single-end reads were generated. The number of reads for each sample was $8 \times 10^{7}$. The number of clean bases of lncRNAs, circRNAs and mRNAs for each sample in filtered data was 12-13 Gb. The error rate of each base in lncRNAs, circRNAs and mRNAs for each sample was $<0.001$. The spliced transcription products of each sample were combined 
Table I. Clinical characteristics of sequencing subjects.

\begin{tabular}{|c|c|c|c|c|c|}
\hline Characteristic & Control $(n=5)$ & Group a $(n=5)$ & Group b $(n=5)$ & Group c $(n=5)$ & P-value \\
\hline Males & 3 & 3 & 3 & 1 & 0.051 \\
\hline Age (years) & $50.40 \pm 7.26$ & $63.60 \pm 5.12$ & $64.40 \pm 6.38$ & $58.60 \pm 5.94$ & 0.010 \\
\hline Height (cm) & $163.00 \pm 9.89$ & $162.40 \pm 8.01$ & $169.20 \pm 5.80$ & $165.80 \pm 5.35$ & 0.482 \\
\hline Weight (kg) & $66.60 \pm 11.28$ & $70.30 \pm 9.91$ & $75.80 \pm 12.37$ & $60.60 \pm 11.26$ & 0.225 \\
\hline Hypertension & 0 & 2 & 1 & 1 & 0.337 \\
\hline Diabetes mellitus & 0 & 0 & 2 & 1 & 0.058 \\
\hline Cancer & 0 & 0 & 1 & 0 & 0.059 \\
\hline
\end{tabular}

Values are expressed as the mean \pm standard error of the mean or $n$. Groups: a, QSBSS patients with coronary heart disease ( $\mathrm{n}=5$ ); $\mathrm{b}, \mathrm{QSBSS}$ patients with chronic gastritis ( $n=5)$; c, QSBSS patients with rheumatoid arthritis ( $n=5)$. QSBSS, Qi stagnation and blood stasis syndrome.
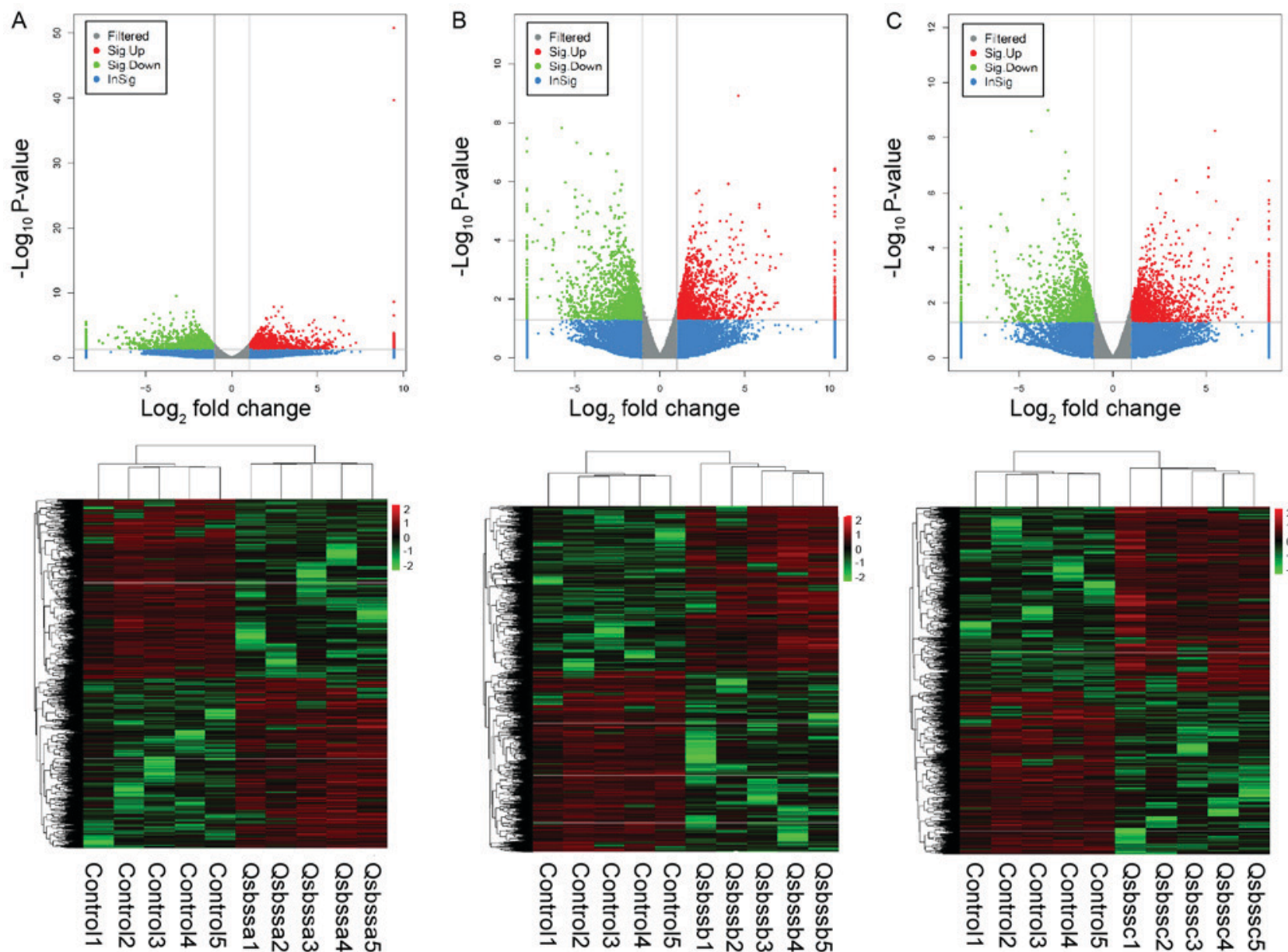

Figure 1. Volcano plot and clustering map of DE lncRNAs. Upper panel, volcano plots indicating up- and down-regulated lncRNAs for the comparison of the normal controls with groups (A) a, (B) b and (C) c. The respective heat maps presenting the hierarchical clustering of DE lncRNAs are displayed in the lower panel. Groups: a, QSBSS patients with coronary heart disease $(n=5)$; b, QSBSS patients with chronic gastritis $(n=5)$; $c$, QSBSS patients with rheumatoid arthritis ( $\mathrm{n}=5)$. QSBSS, Qi stagnation and blood stasis syndrome; IncRNA, long non-coding RNA; DE, differentially expressed.

and screened as lncRNAs with Cuffmerge program in the Cufflinks 2.2.1 software package. All transcripts that overlapped with known mRNAs, other non-coding RNA and non-lncRNA were discarded. Next, the transcripts that longer than 200 bp and $>2$ exons were obtained, and CPC (version 0.9-r2) (26), PLEK (version 1.2) (27), CNCI (version 1.0) (28) and Pfam (version 30) (29) software packages were used to predict transcripts with coding potential. The characteristics (including length, type and number of exons) of lncRNA were analysed after screening by the Cuffcompare program in the Cufflinks 2.2.1 software package (30). CircRNAs were identified using the CircRNA Identifier tool (31). The expression of lncRNAs were calculated using fragments per kb per million reads (FPKM), while the expression of circRNAs 

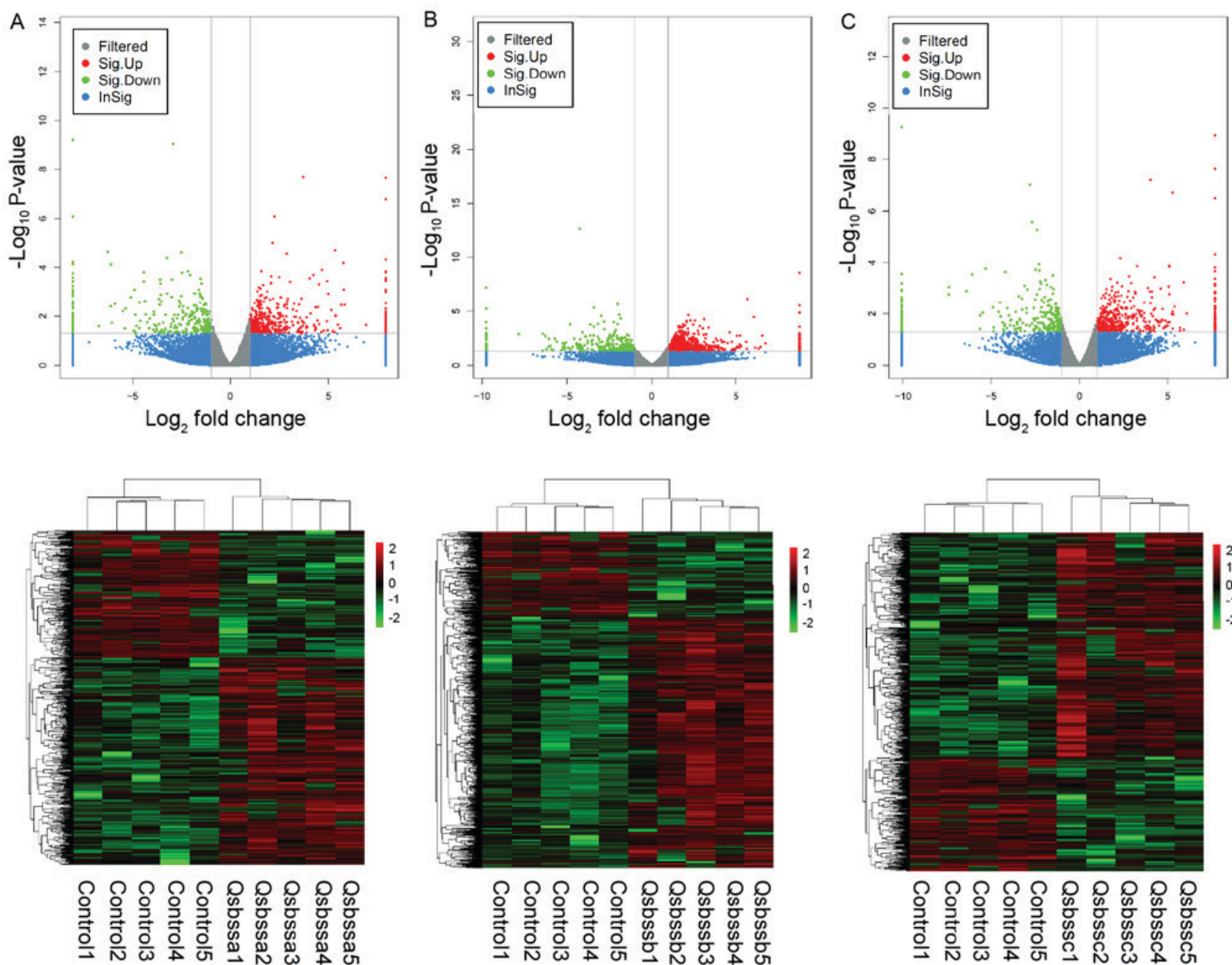

Figure 2. Volcano plot and clustering map of DE circRNAs. Upper panel, volcano plots indicating up- and down-regulated circRNAs for the comparison of the normal controls with groups (A) a, (B) b and (C) c. The respective heat maps presenting the hierarchical clustering of DE circRNAs are displayed in the lower panel. Groups: a, QSBSS patients with coronary heart disease $(n=5)$; b, QSBSS patients with chronic gastritis $(n=5)$; c, QSBSS patients with rheumatoid arthritis $(\mathrm{n}=5)$. QSBSS, Qi stagnation and blood stasis syndrome; circRNA, circular RNA; DE, differentially expressed.

were calculated by spliced reads per million reads (RPM). Then, DESeq2 with negative binomial distribution was used to analyse DE of RNAs (32). All sequencing processes and analyses were performed by Shanghai OE Biotech Co., Ltd. (Shanghai, China).

Gene ontology (GO) annotations and Kyoto encyclopedia of genes and genomes (KEGG) pathway analysis. GO annotations and KEGG pathway analysis were performed to investigate the possible roles of the DE ncRNAs. GO annotations were performed to identify regulatory networks of DE genes (http://geneontology.org). KEGG analysis was also performed to explore the enriched pathways of the DE RNAs based on the KEGG database (http://www.genome.jp/kegg/). To reveal the role and interactions among the DE ncRNAs and mRNAs in QSBSS patients, an ncRNAs regulatory network was constructed using Cytoscape software version 3.2.1 (http://www.cytoscape.org/).

Network pharmacology validation. The process of network pharmacology validation was performed in three steps. First, according to the sequencing results of DE RNAs, the
RNA targets associated with QSBSS were prepared by ID normalization at http://www.uniprot.org/ (33). Next, the active components of Xuefu Zhuyu decoction, a classic and CFDA-approved TCM formula for treating QSBSS, were retrieved from the TCM Systems Pharmacology Database (TCMSP) and the TCM Integrated Database $(34,35)$. The compounds were then screened according to parameters including drug-likeness (DL) and oral bioavailability (OB), and the specific ingredients were selected if their $\mathrm{DL} \geq 0.18$ and $\mathrm{OB}$ $\geq 30 \%$, suggested criteria given by the TCMSP database (34). Finally, the targets of the compounds were predicted using the TCMSP analysis platform and the merged compound-RNA target network was constructed using Cytoscape (version 3.2.1), in order to determine whether any of the DE RNAs associated with QSBSS were targets of the components of Xuefu Zhuyu decoction.

Statistical analysis. The data regarding the clinical characteristics of the participants are expressed as the mean \pm standard error of the mean and the corresponding results were statistically analyzed using one-way analysis of variance. The results of DE RNAs were analyzed by the algorithm of DESeq2 in R 

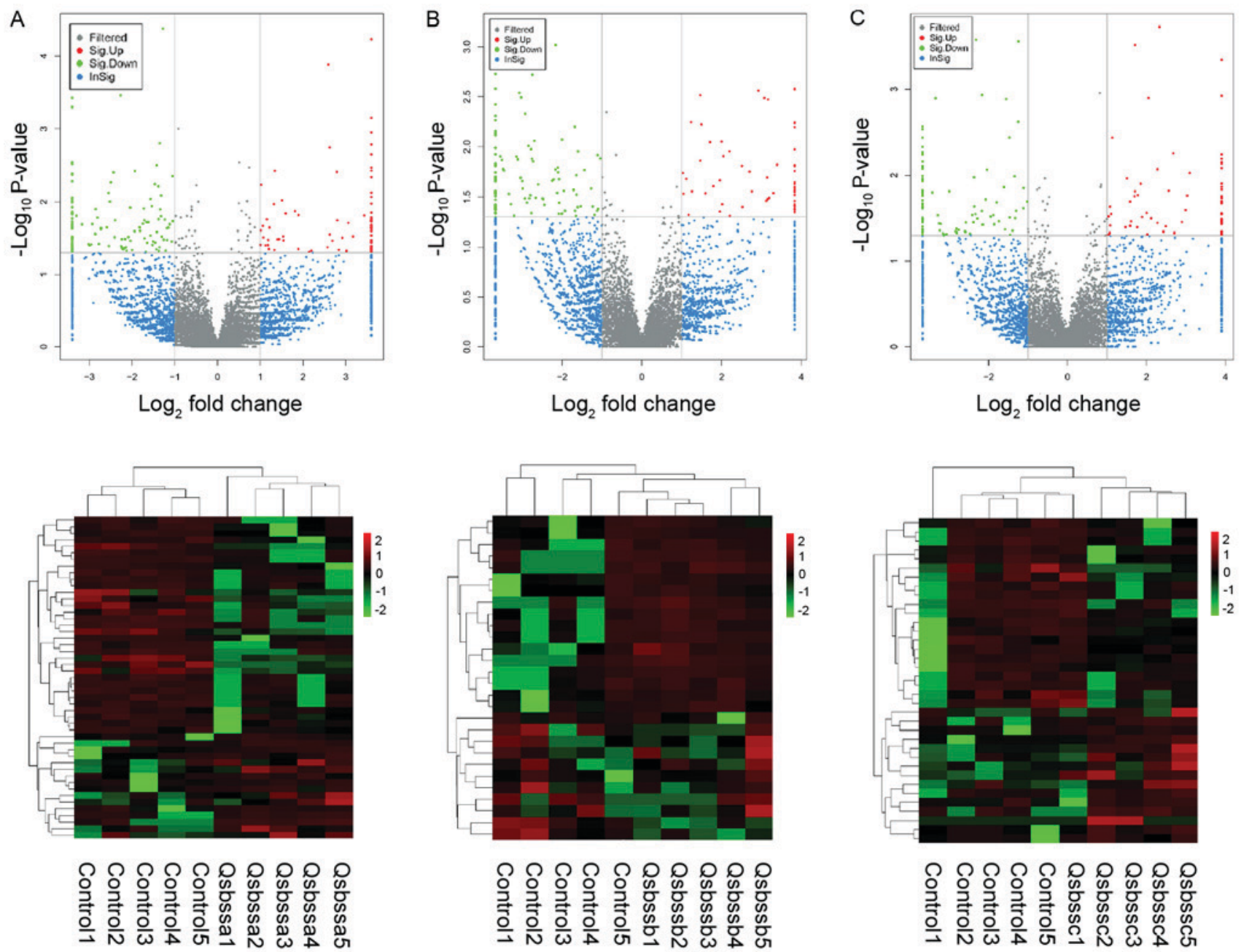

Figure 3. Volcano plot and clustering map of DE mRNAs. Upper panel, volcano plots indicating up- and down-regulated mRNAs for the comparison of the normal controls with groups (A) a, (B) b and (C) c. The respective heat maps presenting the hierarchical clustering of DE mRNAs are displayed in the lower panel. Groups: a, QSBSS patients with coronary heart disease $(n=5)$; $b$, QSBSS patients with chronic gastritis ( $=5$ ); $c$, QSBSS patients with rheumatoid arthritis ( $\mathrm{n}=5)$. QSBSS, Qi stagnation and blood stasis syndrome; DE, differentially expressed.

software (32). The regulatory network for ncRNAs was analyzed by the algorithm of ClueGo in Cytoscape software. $\mathrm{P}<0.05$ was considered to indicate a statistically significant difference.

\section{Results}

Clinical characteristics of participants. A total of 20 participants were recruited for the present study and were divided into 4 groups. The clinical characteristics of these 4 groups were listed in Table I. The 4 groups were matched in terms of sex, as well as body height and weight, and there were no significant differences in terms of the frequency of hypertension, diabetes mellitus and cancer between the groups $(\mathrm{P}>0.05)$.

DE ncRNAs and mRNAs, and their overlap among patient groups with different types of disease. The results of the DE ncRNAs and mRNAs in the comparisons were as follows: A total of 678 DE lncRNAs (448 upregulated and 230 downregulated), 166 DE circRNAs (59 upregulated and 107 downregulated), and 2,676 DE mRNAs (1,287 upregulated and 1,389 downregulated) were identified in group a (CHD with QSBSS vs. controls), respectively. In group b (CG with QSBSS vs. control), 1,158 DE
IncRNAs (901 upregulated and 257 downregulated), $116 \mathrm{DE}$ circRNAs (38 upregulated and 78 downregulated) and 2,880 DE mRNAs (1,336 upregulated and 1,544 downregulated) were detected. A total of 697 DE lncRNAs (470 upregulated and 227 downregulated), 120 DE circRNAs (49 upregulated and 71 downregulated) and 2,776 DE mRNAs (1,420 upregulated and 1,356 downregulated) were identified in group c (RA with QSBSS vs. control). DE ncRNAs (Figs. 1 and 2) and mRNAs (Fig. 3) in the samples were displayed using Volcano plot and clustering maps for groups a, b and c individually. The top 10 upregulated and top 10 downregulated lncRNAs, circRNAs and mRNAs in groups a, b and c are listed in Tables II, III and IV, respectively. Overlapped DE lncRNAs, circRNAs and mRNAs among groups a, b and c were illustrated in a Venn diagram (Fig. 4). A total of 374 up-regulated DE mRNAs, 323 down-regulated DE mRNAs, 81 up-regulated DE lncRNAs, 23 down-regulated DE lncRNAs and 2 down-regulated DE circRNAs overlapped. No up-regulated DE circRNAs overlapped.

Functional prediction of ncRNAs. To further determine the possible functions of the overlapped DE RNAs and investigate the association between the functional groups and 
Table II. Detailed information of the top 10 upregulated and 10 downregulated lncRNAs.

A, Group a

\begin{tabular}{|c|c|c|c|c|c|c|}
\hline Gene ID & Gene name & BM_control & BM_QSBSS & $\log 2$ (fold change) & P-value & Regulation \\
\hline lnc-SUPT16H-3:1 & Novel_lncRNA & 28.45432035 & 374.5259054 & 3.718345886 & $2.06 \times 10^{-8}$ & Up \\
\hline lnc-RALGAPA1-4:1 & Novel_lncRNA & 76.75550691 & 359.25044 & 2.226647757 & $8.13 \times 10^{-7}$ & Up \\
\hline LINC01619:1 & Novel_lncRNA & 44.81014998 & 196.9603127 & 2.136007497 & $1.03 \times 10^{-5}$ & Up \\
\hline lnc-PTP4A2-3:4 & Novel_lncRNA & 7.397430792 & 297.6452117 & 5.330425581 & $2.06 \times 10^{-5}$ & $\mathrm{Up}$ \\
\hline lnc-PKDCC-4:1 & Novel_lncRNA & 13.12539052 & 94.73577234 & 2.851548944 & $2.76 \times 10^{-5}$ & $\mathrm{Up}$ \\
\hline lnc-SOX4-2:1 & Novel_lncRNA & 0.187964496 & 45.41608604 & 7.916599391 & $4.77 \times 10^{-5}$ & Up \\
\hline LINC-PINT:20 & Novel_lncRNA & 0.68761825 & 37.12070593 & 5.7544725 & $6.62 \times 10^{-5}$ & Up \\
\hline lnc-FAN1-6:1 & Novel_lncRNA & 3.007431931 & 76.98804768 & 4.678030497 & $1.25 \times 10^{-4}$ & Up \\
\hline lnc-NEDD9-2:3 & Novel_lncRNA & 204.8829122 & 607.9154915 & 1.569071119 & $1.40 \times 10^{-4}$ & Up \\
\hline lnc-UACA-6:3 & Novel_lncRNA & 2.85167033 & 53.72383561 & 4.235683197 & $2.03 \times 10^{-4}$ & Up \\
\hline lnc-CFLAR-1:1 & Novel_lncRNA & 317.5740647 & 42.23690812 & -2.910516963 & $8.76 \times 10^{-10}$ & Down \\
\hline lnc-C18orf54-3:1 & Novel_lncRNA & 17.59737855 & 0.229176766 & -6.262755929 & $2.35 \times 10^{-5}$ & Down \\
\hline lnc-PTPN6-1:2 & Novel_lncRNA & 73.0536666 & 12.89197843 & -2.502483005 & $2.46 \times 10^{-5}$ & Down \\
\hline lnc-TOB1-4:1 & Novel_lncRNA & 49.503338 & 5.280343401 & -3.228822147 & $4.21 \times 10^{-5}$ & Down \\
\hline lnc-NINJ2-4:3 & Novel_lncRNA & 15.78127325 & 0.229176766 & -6.105609007 & $7.60 \times 10^{-5}$ & Down \\
\hline lnc-VSTM5-1:12 & Novel_lncRNA & 88.5702391 & 19.58027393 & -2.177421065 & $1.42 \times 10^{-4}$ & Down \\
\hline lnc-AC092329.1-1:5 & Novel_lncRNA & 20.45877718 & 0.948506969 & -4.430917734 & $1.61 \times 10^{-4}$ & Down \\
\hline lnc-MDM1-1:10 & Novel_lncRNA & 54.70206769 & 9.823915046 & -2.477225377 & $1.65 \times 10^{-4}$ & Down \\
\hline lnc-CRYBA4-1:53 & Novel_lncRNA & 121.6228189 & 28.76734593 & -2.079909903 & $2.02 \times 10^{-4}$ & Down \\
\hline lnc-PTP4A2-3:3 & Novel_lncRNA & 244.8594302 & 0.916707064 & -8.061277253 & $2.65 \times 10^{-4}$ & Down \\
\hline
\end{tabular}

B, Group b

\begin{tabular}{|c|c|c|c|c|c|c|}
\hline Gene ID & Gene name & BM_control & BM_QSBSS & $\log 2$ (fold change) & P-value & Regulation \\
\hline LINC-PINT:20 & Novel_lncRNA & 0.669136799 & 34.68062215 & 5.695684784 & $7.29 \times 10^{-7}$ & Up \\
\hline lnc-FGL2-3:1 & Novel_lncRNA & 2.497604805 & 1065.79556 & 8.737169794 & $1.34 \times 10^{-5}$ & Up \\
\hline lnc-KIAA0748-2:1 & Novel_lncRNA & 38.88426047 & 178.264157 & 2.196758448 & $2.32 \times 10^{-5}$ & Up \\
\hline lnc-RCAN1-4:1 & Novel_lncRNA & 0.363197716 & 24.31360581 & 6.064864925 & $3.43 \times 10^{-5}$ & $\mathrm{Up}$ \\
\hline lnc-RTEL1.1-3:5 & Novel_lncRNA & 4.653772495 & 40.51149143 & 3.121858609 & $5.39 \times 10^{-5}$ & $\mathrm{Up}$ \\
\hline lnc-INSL4-5:1 & Novel_lncRNA & 19.05513281 & 95.23213668 & 2.321268839 & $6.94 \times 10^{-5}$ & Up \\
\hline lnc-PKDCC-4:1 & Novel_lncRNA & 12.72486822 & 76.09097524 & 2.580074638 & $7.43 \times 10^{-5}$ & Up \\
\hline lnc-RIMS3-5:1 & Novel_lncRNA & 29.60597764 & 126.9453778 & 2.100247466 & $8.52 \times 10^{-5}$ & Up \\
\hline lnc-LYRM7-4:1 & Novel_lncRNA & 36.08770968 & 151.2244038 & 2.067111483 & $8.82 \times 10^{-5}$ & $\mathrm{Up}$ \\
\hline lnc-CTNNA3-3:1 & Novel_lncRNA & 32.24888452 & 139.4355372 & 2.112277136 & $1.02 \times 10^{-4}$ & Up \\
\hline lnc-CFLAR-1:1 & Novel_lncRNA & 307.456305 & 0.353369173 & -9.764989488 & $0.00 \times 10^{-11}$ & Down \\
\hline lnc-APEX1-3:1 & Novel_lncRNA & 211.245845 & 11.0242814 & -4.26016644 & $2.00 \times 10^{-13}$ & Down \\
\hline lnc-PRKAR1A-2:1 & Novel_lncRNA & 1122.360252 & 283.5025529 & -1.985102191 & $2.10 \times 10^{-6}$ & Down \\
\hline lnc-RAG1-5:3 & Novel_lncRNA & 51.27926169 & 4.964879496 & -3.368544883 & $4.78 \times 10^{-6}$ & Down \\
\hline LINC00936:1 & Novel_lncRNA & 963.0937225 & 258.3817153 & -1.89817222 & $2.11 \times 10^{-5}$ & Down \\
\hline lnc-ZNF101-3:1 & Novel_lncRNA & 108.7867263 & 14.13476693 & -2.944182536 & $2.60 \times 10^{-5}$ & Down \\
\hline lnc-MB21D1-3:5 & Novel_lncRNA & 6778.947852 & 1925.503917 & -1.815825315 & $4.63 \times 10^{-5}$ & Down \\
\hline lnc-CCDC111-2:1 & Novel_lncRNA & 87.39175981 & 12.76026571 & -2.775838883 & $9.09 \times 10^{-5}$ & Down \\
\hline lnc-UGDH-3:2 & Novel_lncRNA & 132.0477892 & 28.57958569 & -2.20800324 & $1.00 \times 10^{-4}$ & Down \\
\hline lnc-TOB1-4:1 & Novel_lncRNA & 47.99402748 & 5.477222185 & -3.131338574 & $1.43 \times 10^{-4}$ & Down \\
\hline
\end{tabular}

their underlying mechanisms in the biological networks, the Cytoscape plugin ClueGo was utilized. Significant GO terms in the categories MF and BP were specifically associated with nucleic acid metabolic process, regulation of macromolecule metabolic process, peptidyl-lysine modification and mitotic cell cycle (Figs. 5 and 6). Of note, these DE RNAs coordinate 
Table II. Continued.

C, Group c

\begin{tabular}{|c|c|c|c|c|c|c|}
\hline Gene ID & Gene name & BM_control & BM_QSBSS & $\log 2$ (fold change) & P-value & Regulation \\
\hline lnc-SUPT16H-3:1 & Novel_lncRNA & 28.98663335 & 473.8065463 & 4.030838445 & $6.38 \times 10^{-8}$ & Up \\
\hline lnc-PTP4A2-3:4 & Novel_lncRNA & 7.51850112 & 290.4813765 & 5.271856785 & $1.90 \times 10^{-7}$ & Up \\
\hline PAX8-AS1:6 & Novel_lncRNA & 28.16175293 & 142.8994525 & 2.343191347 & $6.99 \times 10^{-5}$ & Up \\
\hline lnc-ACTR1B-3:18 & Novel_lncRNA & 0.522432354 & 17.71173166 & 5.083317214 & $1.36 \times 10^{-4}$ & $\mathrm{Up}$ \\
\hline lnc-OR4F21-1:1 & Novel_lncRNA & 3.529033861 & 34.29076606 & 3.280474957 & $1.37 \times 10^{-4}$ & Up \\
\hline lnc-ANAPC7-3:1 & Novel_lncRNA & 0.511499328 & 17.62430933 & 5.106690566 & $1.47 \times 10^{-4}$ & Up \\
\hline SNHG4:19 & Novel_lncRNA & 5.177511537 & 36.41675096 & 2.814271445 & $3.52 \times 10^{-4}$ & Up \\
\hline lnc-HACL1-10:1 & Novel_lncRNA & 0.955682697 & 18.22549975 & 4.253282866 & $3.67 \times 10^{-4}$ & Up \\
\hline lnc-LYRM7-4:1 & Novel_lncRNA & 37.9577107 & 131.007337 & 1.787182722 & $4.35 \times 10^{-4}$ & Up \\
\hline lnc-UGT3A2-3:1 & Novel_lncRNA & 1.126190273 & 68.64681004 & 5.92967018 & $5.95 \times 10^{-4}$ & Up \\
\hline TCONS_00066371 & XLOC_034909 & 206.0849162 & 0.190001322 & -10.08301374 & $1.00 \times 10^{-13}$ & Down \\
\hline TCONS_00133003 & XLOC_069024 & 168.4915946 & 24.48540625 & -2.782682585 & $9.50 \times 10^{-8}$ & Down \\
\hline lnc-APEX1-3:1 & Novel_lncRNA & 221.6790465 & 35.26124522 & -2.652317082 & $2.66 \times 10^{-6}$ & Down \\
\hline lnc-GPR123-2:1 & Novel_lncRNA & 145.0012968 & 27.90207696 & -2.377621381 & $5.64 \times 10^{-6}$ & Down \\
\hline lnc-PTPN6-1:2 & Novel_lncRNA & 74.19783961 & 15.26041243 & -2.281583228 & $1.19 \times 10^{-4}$ & Down \\
\hline lnc-MBP-2:6 & Novel_lncRNA & 16.34600311 & 0.412915251 & -5.306948399 & $1.74 \times 10^{-4}$ & Down \\
\hline TCONS_00055344 & XLOC_028836 & 57.55245189 & 10.47564043 & -2.457838946 & $1.91 \times 10^{-4}$ & Down \\
\hline TCONS_00015300 & XLOC_007246 & 68.91323715 & 14.48951949 & -2.249771377 & $2.09 \times 10^{-4}$ & Down \\
\hline lnc-SPG7-1:6 & Novel_lncRNA & 20.96867059 & 1.140007934 & -4.201119628 & $2.34 \times 10^{-4}$ & Down \\
\hline TCONS_00113555 & XLOC_059030 & 13.52330416 & 0.199388024 & -6.083725124 & $2.93 \times 10^{-4}$ & Down \\
\hline
\end{tabular}

Comparison A (Group a vs. Group control), B (Group b vs. Group control) and C (Group c vs. Group control). Groups: a, QSBSS patients with coronary heart disease $(n=5)$; b, QSBSS patients with chronic gastritis $(n=5)$; c, QSBSS patients with rheumatoid arthritis $(n=5)$. lncRNA, long non-coding RNA; QSBSS, Qi stagnation and blood stasis syndrome; BM, base mean.

with each other to perform their functions and the relevant major biochemical pathways and signal transduction pathways were predicted by enrichment analysis based on the KEGG database. A scatterplot of the DE RNAs was employed to demonstrate the KEGG enrichment analysis. In this plot, the degree of KEGG enrichment is assessed by the enrichment factor, P-value and number of genes. A greater enrichment factor is associated with a P-value closer to zero and a greater number of genes, and therefore a more significantly enriched pathway. When the data were analyzed regarding the co-expression of genes of DE lncRNAs, DE mRNAs and target genes of DE circRNAs, the most significantly enriched KEGG pathways were sphingolipid, neurotrophin, AMP-activated protein kinase (AMPK), mRNA surveillance pathway and endocytosis (Fig. 7). These major BPs and signal transduction pathways provide insight regarding further directions of research on the biological basis of QSBSS.

Merged network of DE RNAs and target fishing. As presented in the network for compounds and targets (Fig. 8) and the merged network (Fig. 9), the targets of active components of Xuefu Zhuyu decoction partly covered the targets in the DE RNAs of QSBSS discovered by sequencing. The shared targets of QSBSS and Xuefu Zhuyu Decoction were interleukin $1 \beta$, DNA topoisomerase I, potassium voltage-gated channel subfamily $\mathrm{H}$ member 2 , nuclear receptor coactivator 1 , mitogen-activated protein kinase 8 , insulin-like growth factor 1 receptor and calcium/calmodulin-dependent protein kinase kinase 2.

\section{Discussion}

In the present study, expression profiles of lncRNAs, circRNAs and mRNA were obtained by next-generation sequencing and compared in patients with QSBSS and three different types of disease (CHD, CG or RA vs. normal controls). A total of 104 lncRNAs, 2 circRNAs and 697 mRNAs were finally identified to be significantly DE among groups $\mathrm{a}, \mathrm{b}$ and $\mathrm{c}$ in a Venn diagram. These DE RNAs were subsequently displayed in a Volcano Plot and integrated into hierarchical categories according to heat maps and hierarchical clustering. GO and KEGG analyses were used to predict their relevant functions by ClueGo. A network pharmacology study was also performed to preliminarily confirm the DE RNAs with the hypothesis that the DE RNAs of QSBSS may be regulated by the TCM herbs with efficacy in treating QSBSS. These DE RNAs and their corresponding pathways may be considered highly relevant to QSBSS, and may contribute to the explanation of Zheng and prediction of therapeutic targets of the TCM formula.

QSBSS is a common Zheng that is thought to be associated with the manifestation of numerous types of disease, 


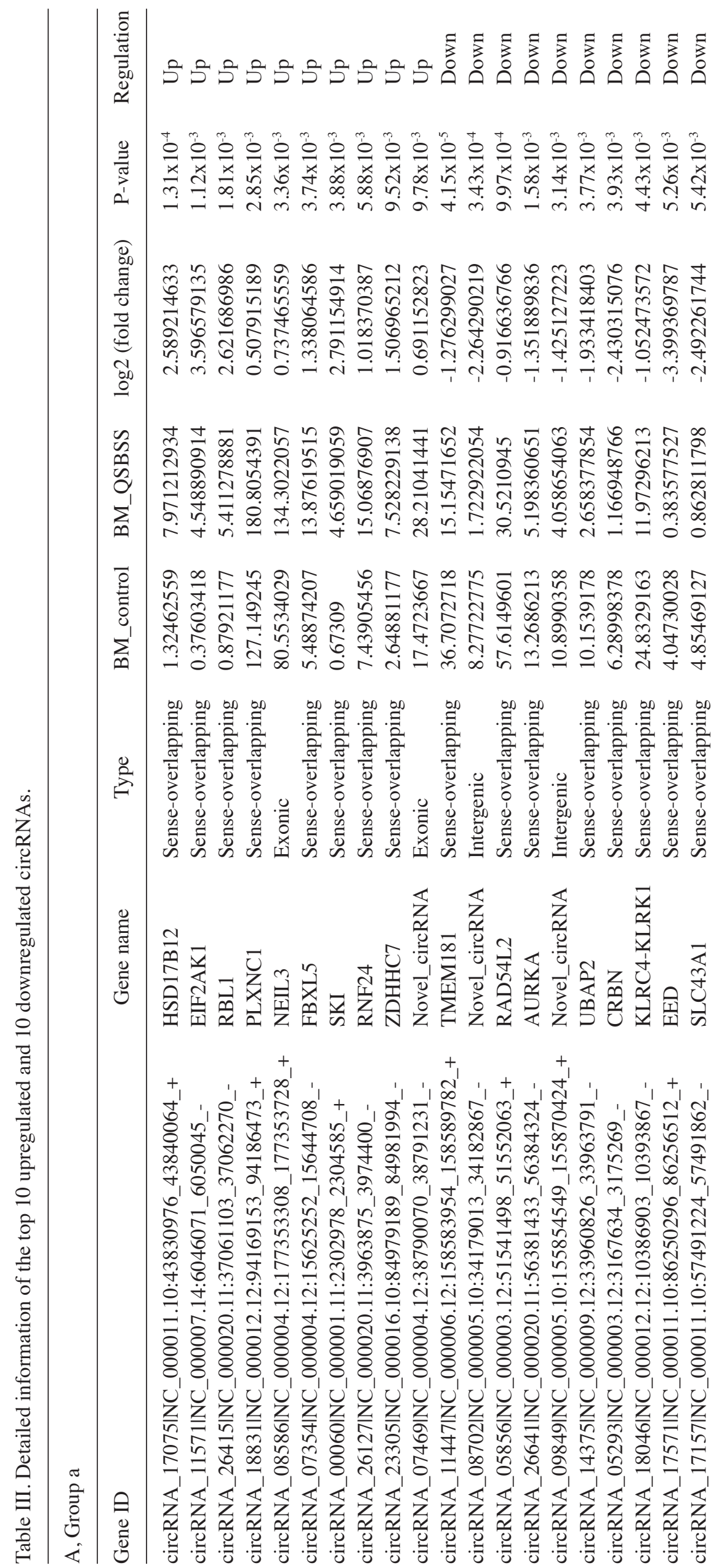

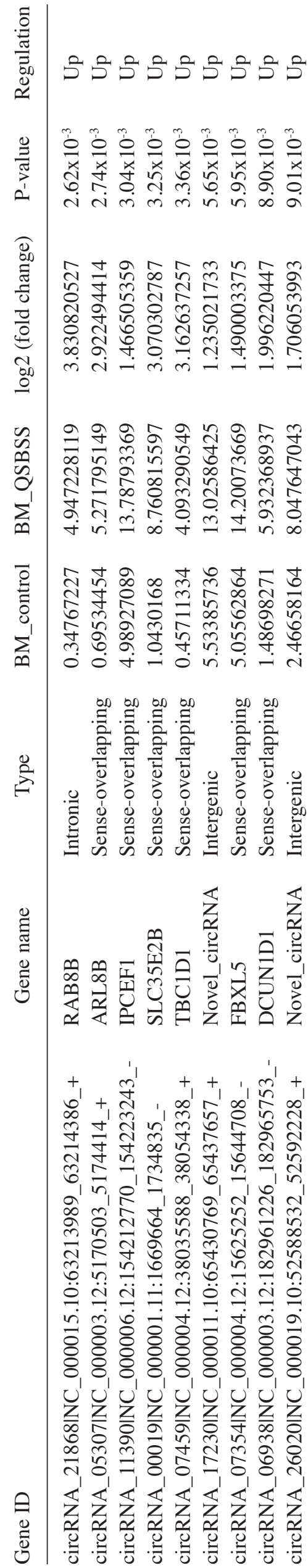




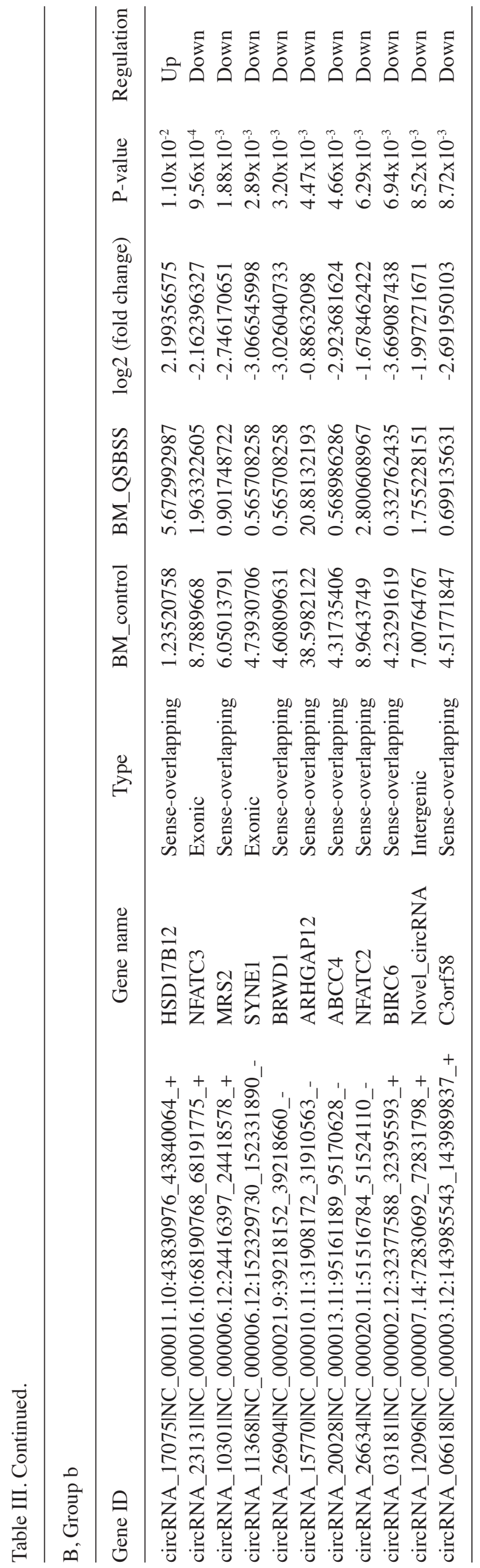

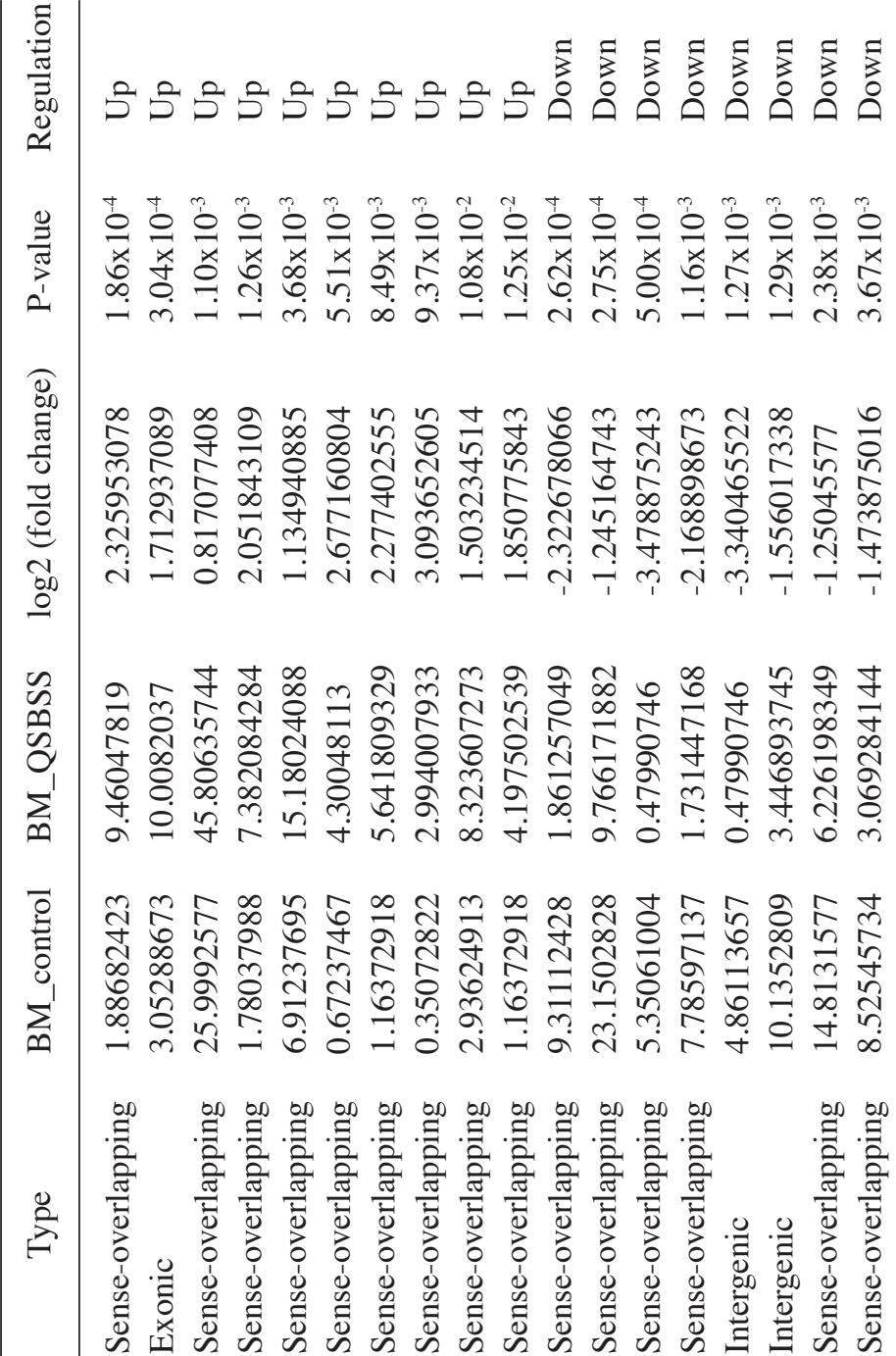

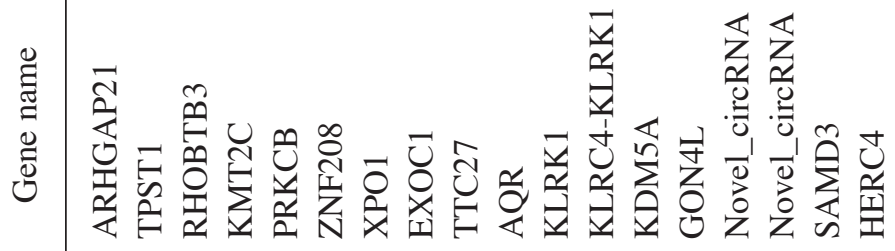

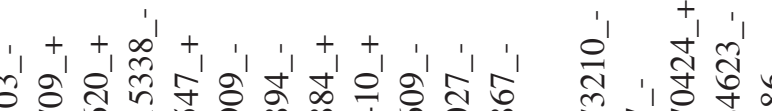
유융

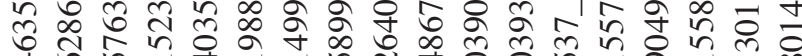

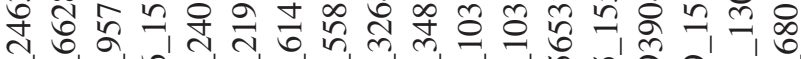

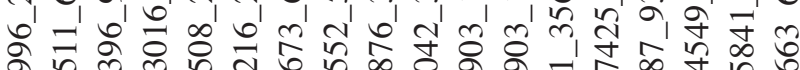
ลे चु त్రీ ป

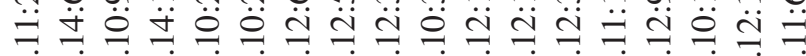

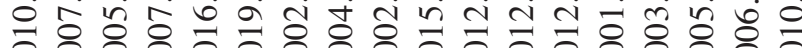
$88088088 \% 88 \% 8080$

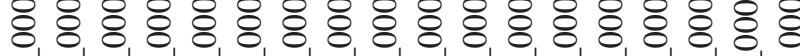

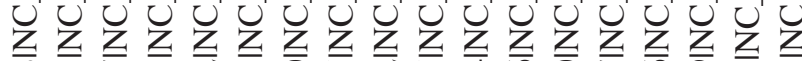

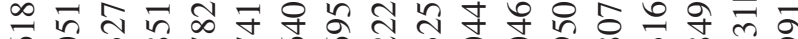

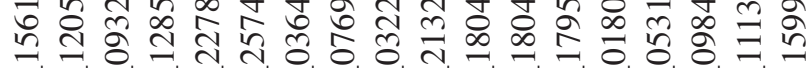

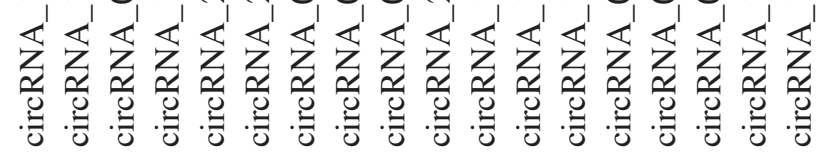




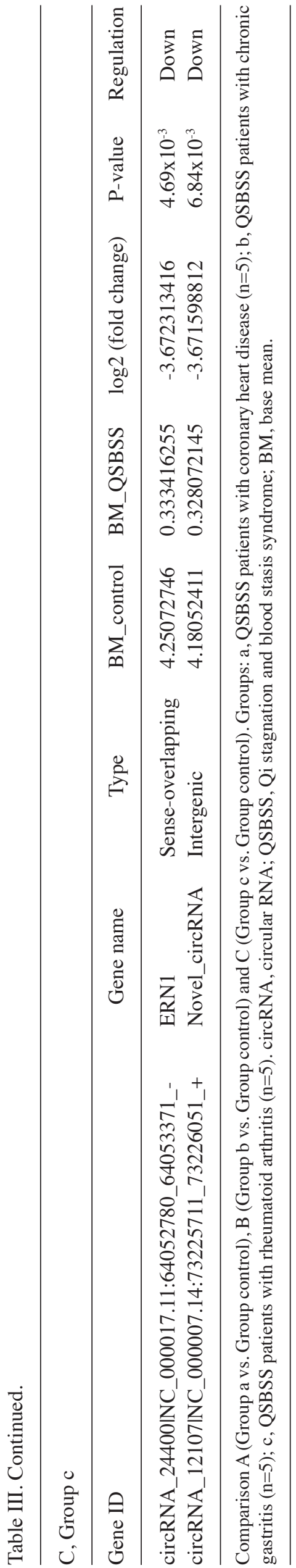

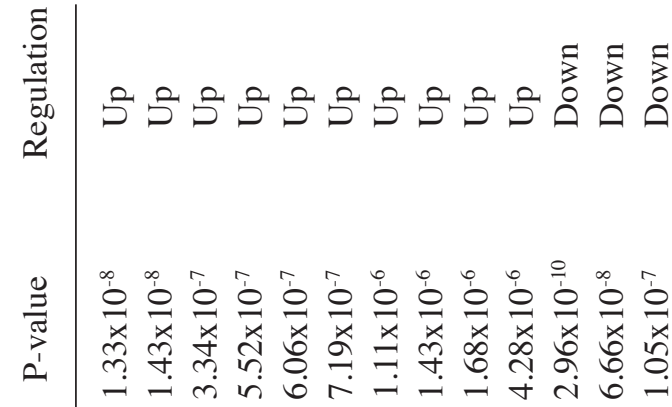

웅 రิ山े ते

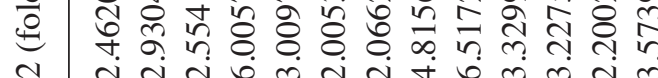

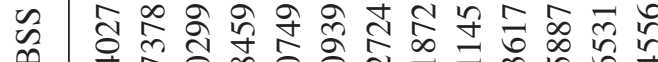

类

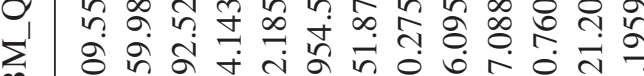

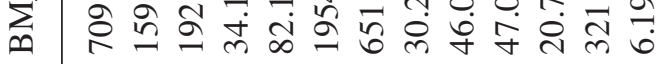

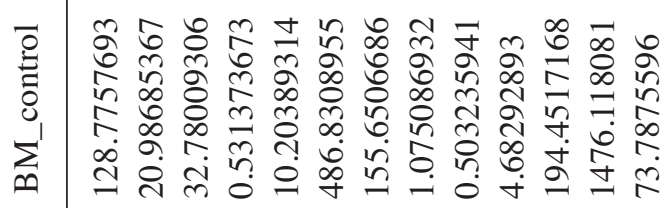

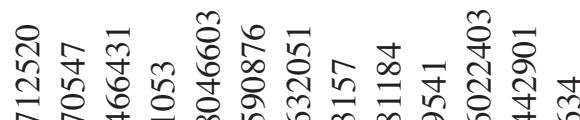

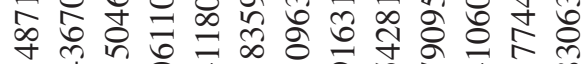

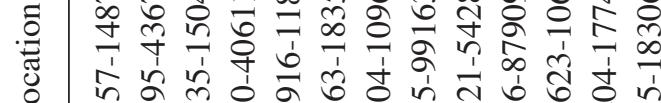

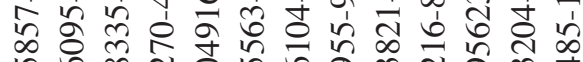

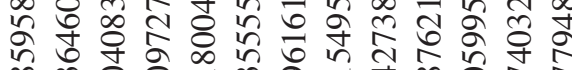
年守

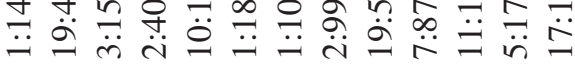

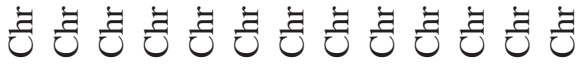

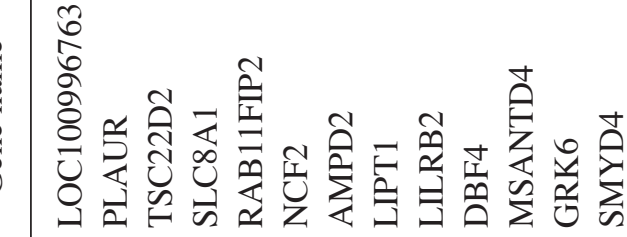

它

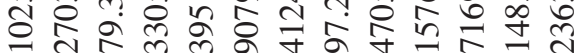

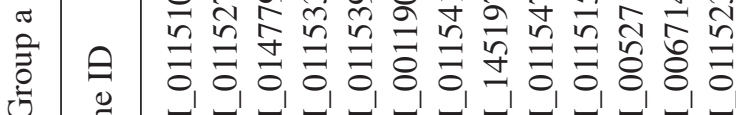

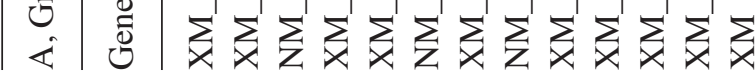



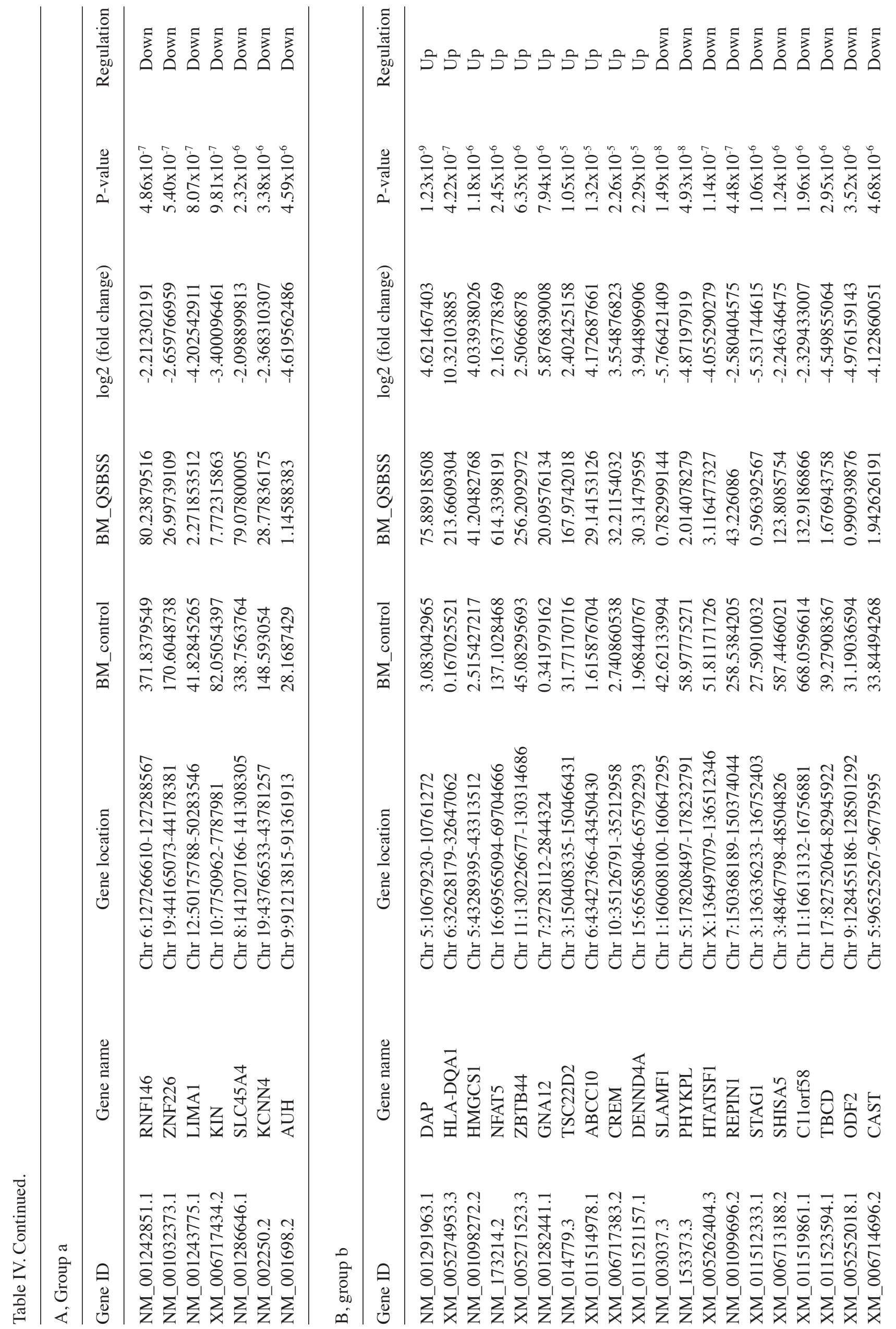


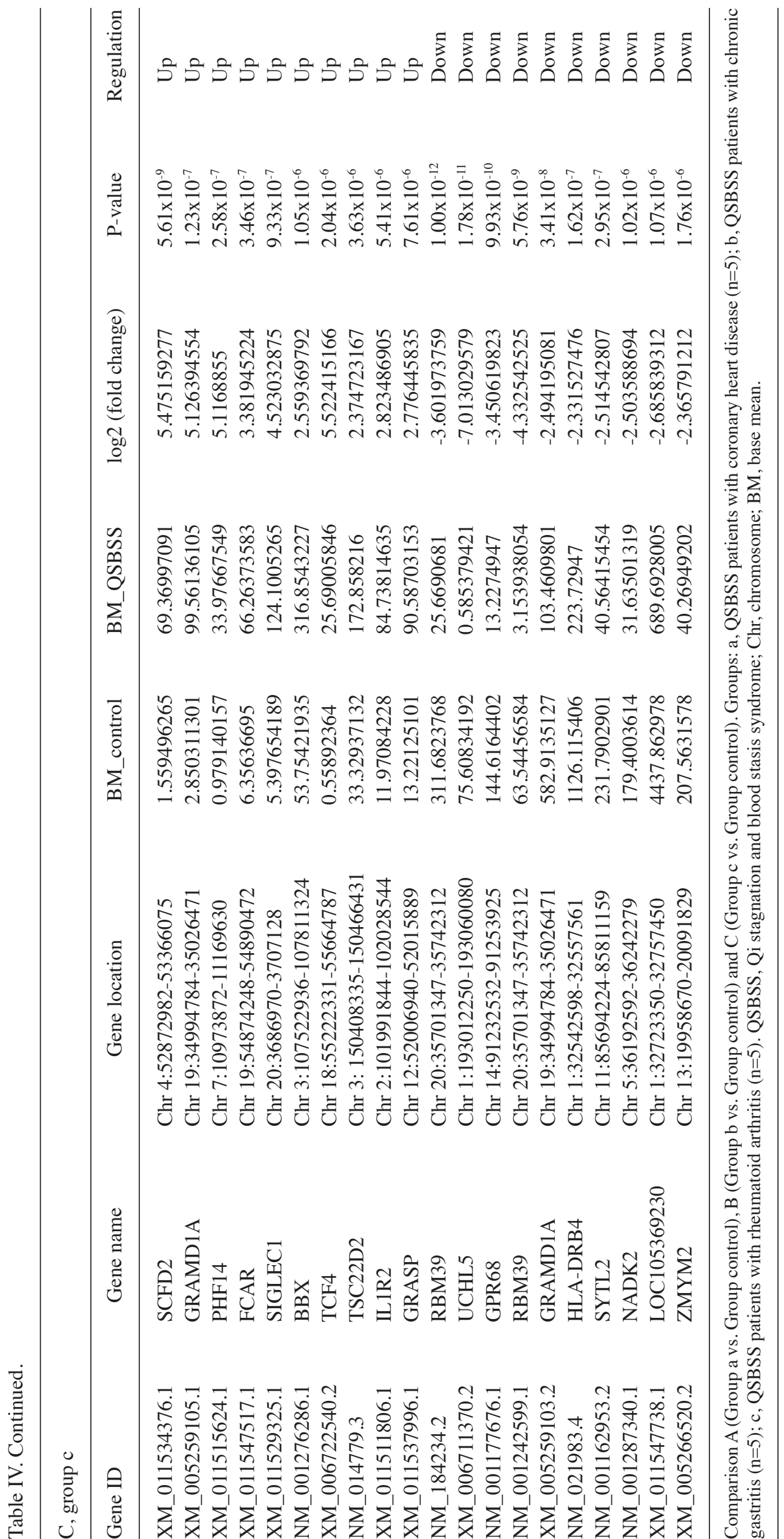




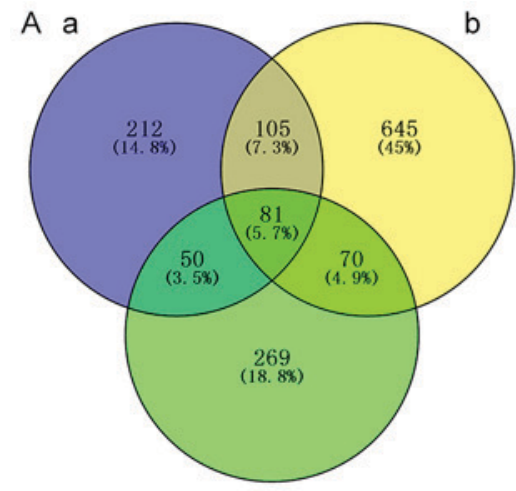

C

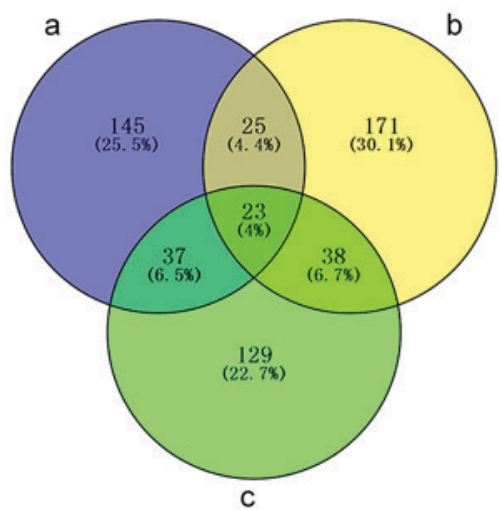

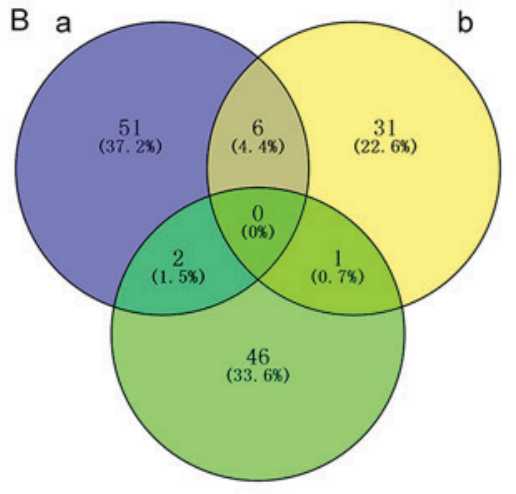

C

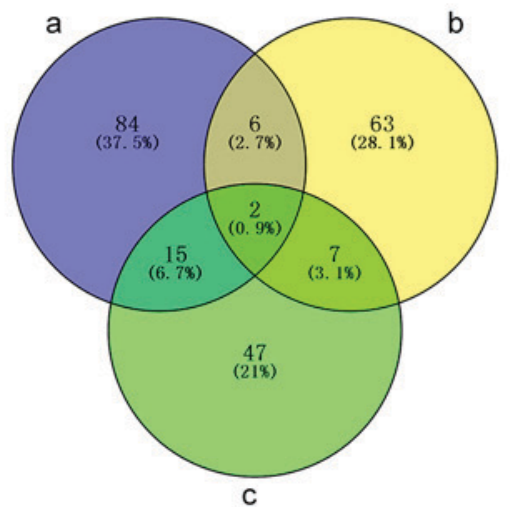

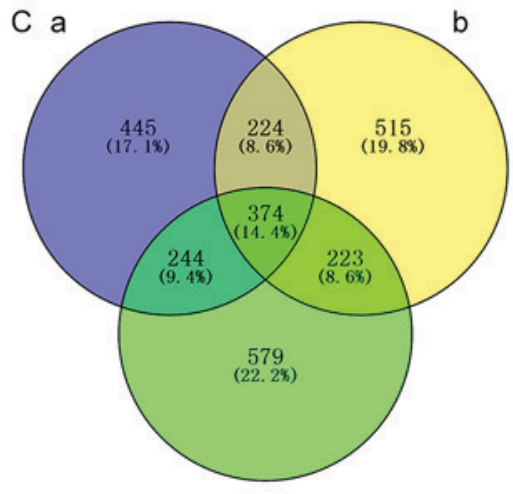

C

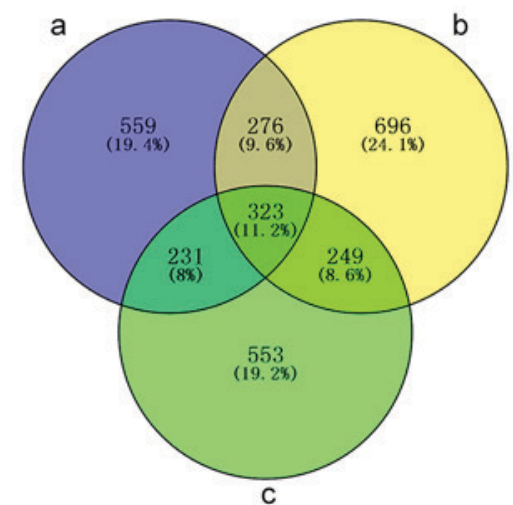

Figure 4. Venn diagram of overlapped DE lncRNAs, circRNAs and mRNAs. Venn diagrams displaying the number of overlapped up-regulated (upper panel) and down-regulated (lower panel) DE (A) lncRNAs, (B) circRNAs and (C) mRNAs among groups a, b and c. Groups: (a) QSBSS patients with coronary heart disease ( $\mathrm{n}=5$ ); (b) QSBSS patients with chronic gastritis ( $\mathrm{n}=5$ ); (c) QSBSS patients with rheumatoid arthritis (n=5). QSBSS, Qi stagnation and blood stasis syndrome; lncRNA, long non-coding RNA; circRNA, circular RNA; DE, differentially expressed.

including CHD, CG and RA, and it is always used to define the condition of unsmooth flow of Qi and blood (36). In addition to distending pain in a fixed place, one symptom of QSBSS is emotional problems, including irritability and depression (21). The development of QSBSS is complex (37) and the mechanism remains largely elusive. The competing endogenous (ce)RNA hypothesis posits that miRNA may bind to an argonaute protein and form the miRNA-guided RNA-induced silencing complex (RISC) to target the relevant mRNA, leading to accelerated degradation, blocked translation and decreased expression, while ceRNA with shared target sites competes for miRNA targeting and binding in the RISC, thus sequestering the miRNA activity, resulting in repression of the expression the target gene of the respective miRNA (14). Furthermore, lncRNA, circRNA and pseudogene transcripts have gained considerable attention as they may work together to regulate ceRNA mechanisms. Despite the growing interest in exploring the biological basis of Zheng from DNA and RNA (38-41), the molecular mechanisms underlying the interaction of ncRNAs and mRNAs in QSBSS have remained largely elusive. Therefore, for the first time, the present study provided and compared expression profiles of lncRNAs, circRNAs and mRNAs in QSBSS obtained by next-generation sequencing. These pioneering discoveries may deepen the current understanding of the mechanisms of Zheng as well as provide a foundation for further scientific research into the molecular basis of TCM theory.
Previous studies have indicated the links between QSBSS and the processes of inflammation, immune response and sympathetic regulation (42-44). Of note, glycoprotein containing disulfide bonds, $\mathrm{G}$ protein-coupled receptor signaling pathways and catecholamine neurotransmitter activity were identified to constitute the biological basis of QSBSS through methods of data mining, which integrated the results of nearly all of the previous research on QSBSS (45). Another previous study suggested that QSBSS patients suffered from an imbalance of lower pneumogastric nerve activity and higher sympathetic nerve activity, as indicated by a heart rate variability analysis (43). Although evidence for the biological basis of QSBSS has accumulated, no comprehensive analysis of the ncRNA and mRNA profile in QSBSS has been performed to date, to the best of our knowledge. Thus, next-generation sequencing was applied in the present study to analyze DE ncRNAs and mRNAs in patients with QSBSS.

Bioinformatics analysis of the RNA sequencing results of the present study indicated that DE RNAs in QSBSS were most significantly enriched in pathways including the sphingolipid signaling pathway, the neurotrophin signaling pathway, AMPK and endocytosis. These results imply that QSBSS is not only associated with an imbalance of neural function, but also with a deregulation of energy metabolism. Specifically, sphingomyelin and its metabolic products are known to have second messenger functions in a variety of cellular signaling pathways $(46,47)$. Among its metabolic products, ceramide 


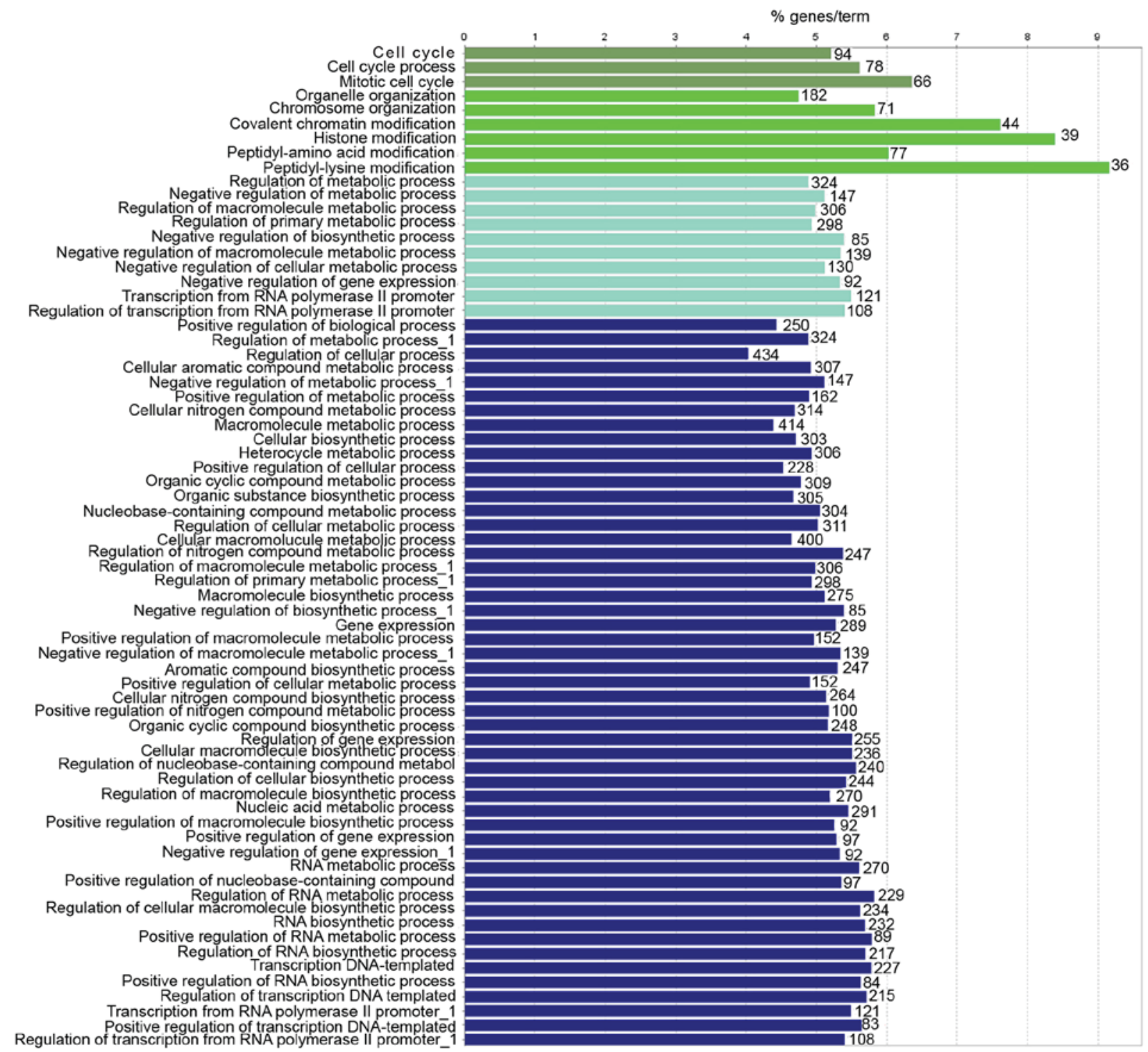

Figure 5. Analysis of DE RNAs by ClueGo. The enrichment analysis of DE RNAs was performed using ClueGo and the most significant terms are presented, where a higher score indicates a higher number of genes involved. The same color indicates that it was possible to cluster the respective terms. DE, differentially expressed.

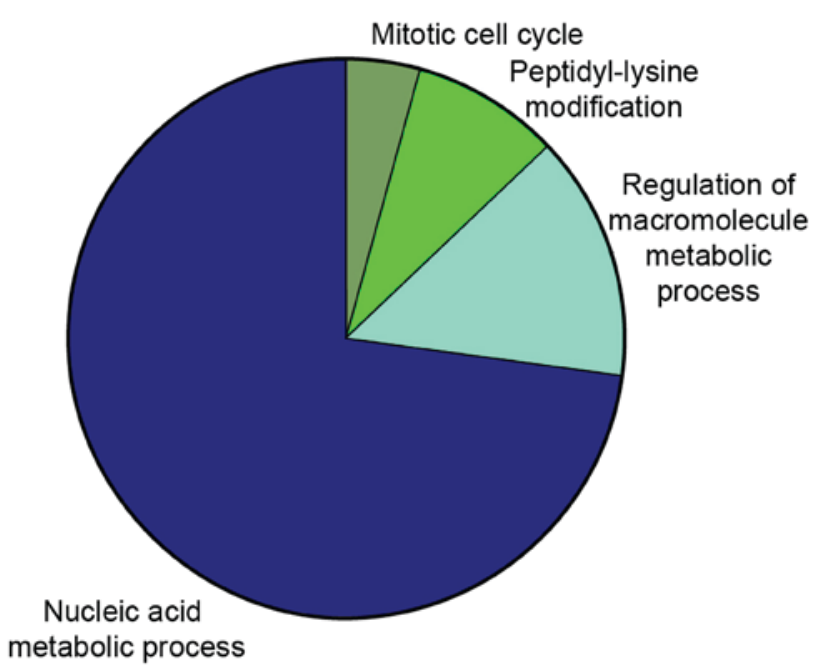

Figure 6. GO analysis of DE RNAs. The GO enrichment analysis of DE RNAs is represented by the pie charts. GO, gene ontology; DE, differentially expressed. and S1P regulate cellular responses to stress, with generally opposing effects $(48,49)$. Neurotrophins are a family of trophic factors involved in the differentiation and survival of neural cells, which are central for the development of diseases including hereditary sensory and autonomic neuropathy, as well as congenital pain insensitivity with anhidrosis (50). Endocytosis is a mechanism for cells to remove ligands, nutrients, plasma membrane proteins and lipids from the cell surface, which is essential for the advance of hereditary sensory and autonomic neuropathy (51). AMPK is a serine threonine kinase that is highly conserved through evolution and the AMPK system acts as a sensor of the cellular energy status $(52,53)$. These functions of enriched pathways may be indicative of why QSBSS frequently manifests as pain accompanied with obscure sensations, including a distending and tingling sensation, as well as emotional problems, including irritability and depression (17). Of note, QSBSS is a stagnation of Qi and the flow of blood, which is known to carry the substance, including oxygen and energy in the body (54). 


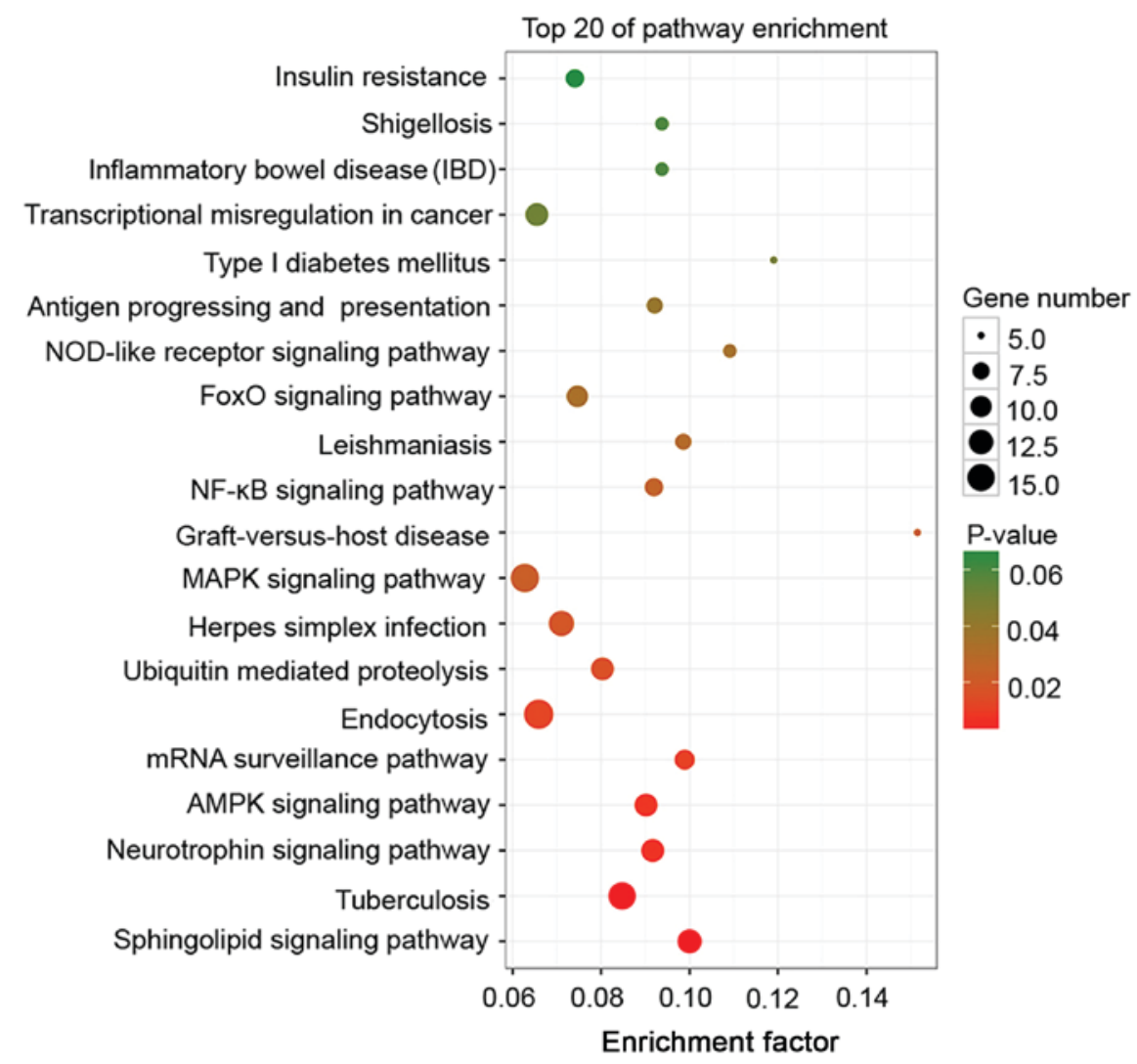

Figure 7. Enriched Kyoto Encyclopedia of Genes and Genomes pathways. The pathway scatterplot displays the statistics of the pathway enrichment of the differentially expressed RNAs. MAPK, mitogen-activated protein kinase; NF, nuclear factor; Fox, forkhead box; AMPK, 5'AMP-activated protein kinase; NOD, nucleotide-binding oligomerization domain.

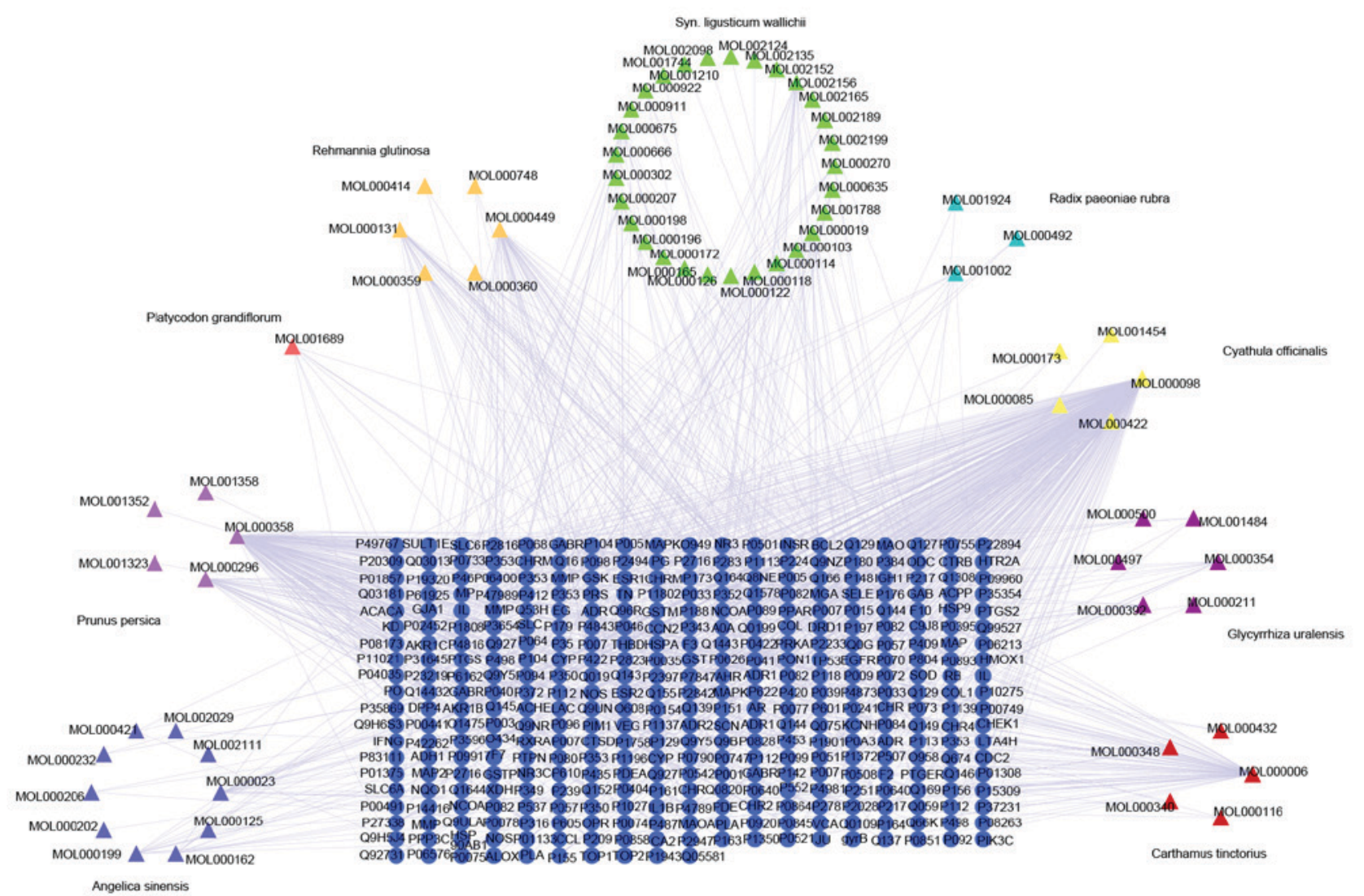

Figure 8. Putative targets for Xuefu Zhuyu decoction, a treatment for Qi stagnation and blood stasis syndrome. The triangular nodes represent the screened ingredients and the circular nodes represent the predicted targets. 


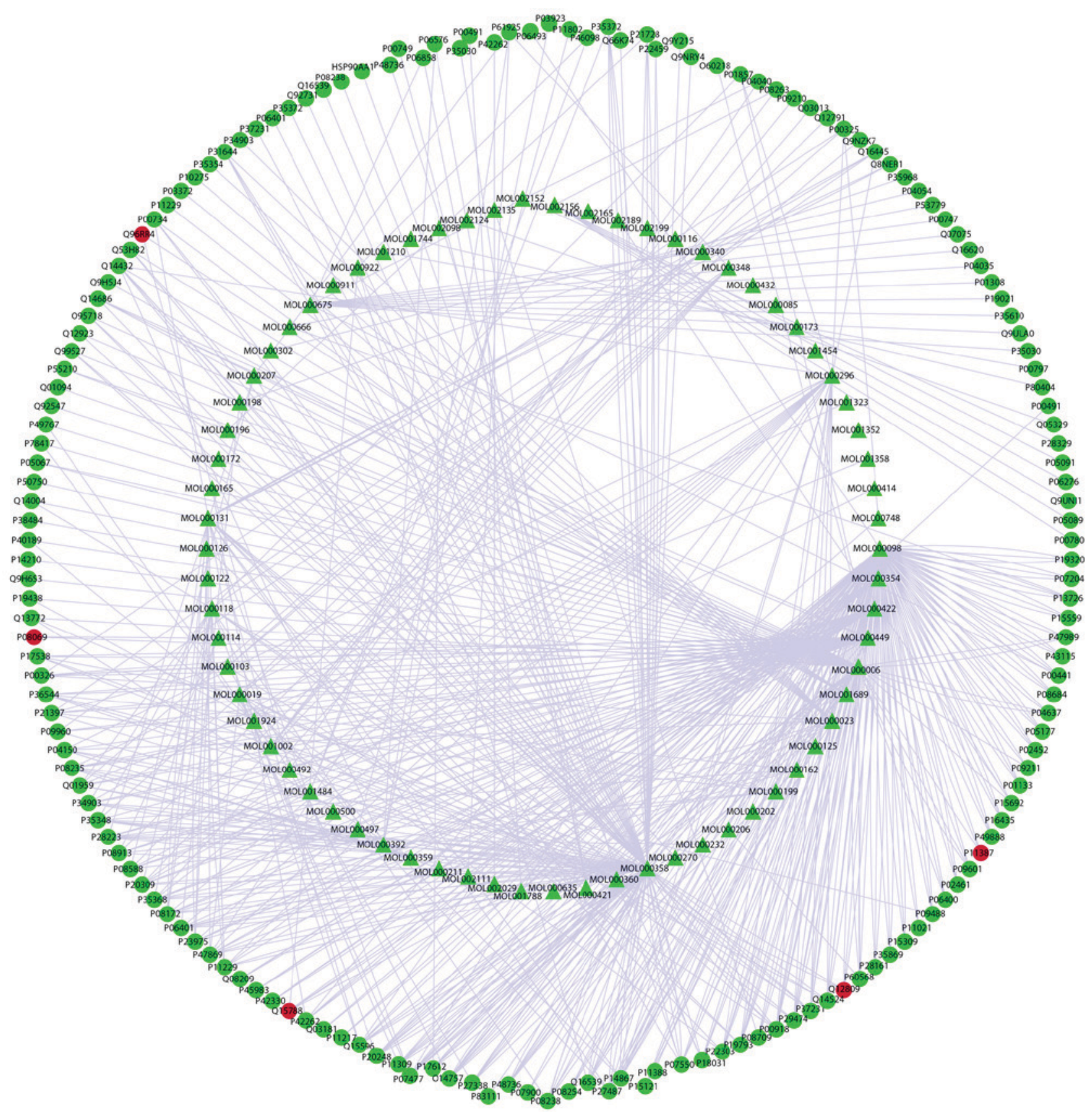

Figure 9. Network of the targets of Xuefu Zhuyu decoction (treatment for QSBSS) and differentially expressed RNAs in QSBSS. The green triangular nodes represent active components, the green circular nodes represent targets and the red nodes represent shared targets of QSBSS and Xuefu Zhuyu Decoction. QSBSS, Qi stagnation and blood stasis syndrome.

Hence, it is likely that the biological basis of QSBSS may be directly associated with a dysfunction of energy metabolism and the autonomic nervous system, which requires more in-depth study in the future.

To the best of our knowledge, the present study was the first to explore the biological basis of Zheng in subjects with different diseases rather than different Zheng in one disease and it was attempted to interpret QSBSS, one of the common Zheng, from the perspective of ncRNAs and mRNAs, which were determined by next-generation sequencing technology. It is worth mentioning that the grouping design in the present study may be implemented to assess any type of Zheng in further studies. However, one of the limitations of the present study is the lack of validation of DE RNAs by quantitative (q)PCR as the cohort size was very small and qPCR analysis was not performed, which is likely to be resolved in a future study. Besides, the inter-group and inter-patient variability should also be analysed in the future study. Furthermore, one intended utility of these DE RNAs and enriched pathways is to predict the potential targets of TCM formulae that treating QSBSS, which mainly include interleukin $1 \beta$, DNA topoisomerase I and potassium voltage-gated channel subfamily $\mathrm{H}$ member 2 . These putative targets may be candidates for experimental validation of the mechanisms of action of the TCM formulae, which may be essential for the optimization of ancient formulae and the discovery of novel drugs. 


\section{Acknowledgements}

Not applicable.

\section{Funding}

This work was supported by the China Food and Drug Administration Project (grant no. 201207009).

\section{Availability of data and materials}

The datasets used and analyzed during the current study are available from the corresponding author on reasonable request.

\section{Authors' contributions}

GC designed the present study, and analyzed and interpreted the data. JLG, HQH, CL, YML, JL and JW enrolled the participants and examination of the blood samples. GC was a major contributor in writing the manuscript. All authors read and approved the final manuscript.

\section{Ethical approval and consent to participate}

The protocol of the present study was approved by the Ethics Committee of Guang'anmen Hospital (Beijing, China). Informed consent was provided by all of the participants.

\section{Patient consent for publication}

Not applicable.

\section{Competing interests}

The authors declare that they have no competing interests.

\section{References}

1. Yuan R and Lin Y: Traditional Chinese medicine: An approach to scientific proof and clinical validation. Pharmacol Ther 86 191-198, 2000.

2. Balta S, Cakar M, Demırkol S, Kucuk U, Ay SA and Unlu M: May chest pain describe coronary heart disease? Croat Med J 54: 411,2013

3. Wang C, Niimi M, Watanabe T, Wang Y, Liang J and Fan J: Treatment of atherosclerosis by traditional Chinese medicine: Questions and quandaries. Atherosclerosis 277: 136-144, 2018.

4. Zha LH, He LS, Lian FM, Zhen Z, Ji HY, Xu LP and Tong XL: Clinical strategy for optimal traditional chinese medicine (TCM) herbal dose selection in disease therapeutics: Expert consensus on classic TCM herbal formula dose conversion. Am J Chin Med 43: 1515-1524, 2015 .

5. Wang YH and Xu AL: Zheng: A systems biology approach to diagnosis and treatments. Sci Art Sci Traditional Med (Suppl): S13-S15, 2014

6. Blázovics A: The interpretation and integration of traditional Chinese phytotherapy into Western-type medicine with the possession of knowledge of the human genome. Orv Hetil 159: 696-702, 2018 (In Hungarian).

7. Josephine PB: A global scientific challenge: Learning the right lessons from ancient healing practices. Sci Art Scie Traditional Med (Suppl) S7-S9, 2014.

8. Chen G, Xue WD and Zhu J: Full genetic analysis for genome-wide association study of Fangji: A powerful approach for effectively dissecting the molecular architecture of personalized traditional Chinese medicine. Acta Pharmacol Sin 39: 906-911, 2018
9. Gao JL, Chen G, He QY, Li J and Wang J: Analysis of chinese patent medicine prescriptions for qi stagnation and blood stasis syndrome. Zhongguo Zhong Yao Za Zhi 42: 187-191, 2017 (In Chinese).

10. Li Y, Li R, Ouyang Z and Li S: Herb Network Analysis for a Famous TCM Doctor's prescriptions on treatment of rheumatoid arthritis. Evid Based Complement Alternat Med 2015: 451319, 2015.

11. Liu C, Chen G, Liu D, Nie S, Wang X, Huang X, Li H, Li Y, Luo S, Zhao G, et al: Observational study of the association between TCM zheng and types of coronary artery stenosis: Protocol of a multicenter case series study. Evid Based Complement Alternat Med 2018: 2564914, 2018.

12. Guttman M and Rinn JL: Modular regulatory principles of large non-coding RNAs. Nature 482: 339-346, 2012.

13. Gilbert WV, Bell TA and Schaening C: Messenger RNA modifications: Form, distribution, and function. Science 352: 1408-1412, 2016.

14. Salmena L, Poliseno L, Tay Y, Kats L and Pandolfi PP: A ceRNA hypothesis: The Rosetta Stone of a hidden RNA language? Cell 146: 353-358, 2011.

15. Thomson DW and Dinger ME: Endogenous microRNA sponges: Evidence and controversy. Nat Rev Genet 17: 272-283, 2016.

16. Memczak S, Jens M, Elefsinioti A, Torti F, Krueger J, Rybak A, Maier L, Mackowiak SD, Gregersen LH, Munschauer M, et al: Circular RNAs are a large class of animal RNAs with regulatory potency. Nature 495: 333-338, 2013.

17. Liu J, Liu T, Wang $X$ and He A: Circles reshaping the RNA world: From waste to treasure. Mol Cancer 16: 58, 2017.

18. Hopkins AL: Network pharmacology: The next paradigm in drug discovery. Nat Chem Biol 4: 682-690, 2008.

19. Li S and Zheng B: Traditional Chinese medicine network pharmacology: Theory, methodology and application. Chin J Nat Med 11: 110-120, 2013.

20. Zheng XY (eds): Guiding Principles for Clinical Research of New Drugs in Traditional Chinese Medicine (Trial) 2002. Vol 2. $1^{\text {th }}$ edition. China Med Sci Technol Press, BeiJing, BJ, pp69-126, 2002.

21. Wang J, Gao JL, Chen G and Haoqiang H: Diagnosis criteria on Qi Stagnation and Blood Stasis Syndrome. Chin J Experimental Traditional Med Formulae 24: 16-20, 2018 (In Chinese).

22. Shen CT, Zhang L, Wang Z, Chen QG, Chen BW, Yuan Y, Zhong Y, Zhu J, Chen Y and Wang YY: Standard for symptoms and signs of Traditional Chinese Medicine in new drug clinical trials. J Chin Med 54: 1265-1267, 2013.

23. American College of Cardiology Foundation/American Heart Association: 2012 ACCF/AHA/ACP/AATS/PCNA/SCAI/STS guideline for the diagnosis and management of patients with stable ischemic heart disease. J Am College Cardiololy 60: 2564-603, 2012.

24. Rheumatology Branch of Chinese Medical Association: The diagnosis and treatment for rheumatoid arthritis. Chin J Rheumatol 14: 265-269, 2010 (In Chinese).

25. Fang JY, Liu WZ, Li ZS, Du YQ, Ji XL, Ge ZZ, Li YQ, Ni JM, Lv $\mathrm{NH}, \mathrm{Wu}$ KC, et al: Digestive Disease Branch of Chinese Medical Association: Consensus on chronic gastritis in China (Shanghai 2012). Zhong Guo Yi Xue Qian Yan Za Zhi 5: 44-55, 2013.

26. Kong L, Zhang Y, Ye ZQ, Liu XQ, Zhao SQ, Wei L and Gao G: CPC: Assess the protein-coding potential of transcripts using sequence features and support vector machine. Nucleic Acids Res 35: W345-W349, 2007.

27. Li A, Zhang J and Zhou Z: PLEK: A tool for predicting long non-coding RNAs and messenger RNAs based on an improved k-mer scheme. BMC Bioinformatics 15: 311, 2014.

28. Sun L, Luo H, Bu D, Zhao G, Yu K, Zhang $C$ and Zhao Y: Utilizing sequence intrinsic composition to classify protein-coding and long non-coding transcripts. Nucleic Acids Res 41: e166, 2013.

29. Finn RD, Bateman A, Clements J, Coggill P, Eberhardt RY, Eddy SR, Heger A, Hetherington K, Holm L, Mistry J, et al: Pfam: The protein families database. Nucleic Acids Res 42 (Database Issue): D222-D230, 2014.

30. Ghosh S and Chan CK: Analysis of RNA-Seq data using TopHat and Cufflinks. Methods Mol Biol 1374: 339-361, 2016.

31. Gao Y, Wang J and Zhao F: CIRI: An efficient and unbiased algorithm for de novo circular RNA identification. Genome Biol 16: 4, 2015.

32. Love MI, Huber W and Anders S: Moderated estimation of fold change and dispersion for RNA-seq data with DESeq2. Genome Biol 15: 550, 2014 
33. Apweiler R, Bairoch A, Wu CH, Barker WC, Boeckmann B, Ferro S, Gasteiger E, Huang H, Lopez R, Magrane M, et al: UniProt: The universal protein knowledgebase. Nucleic Acids Res 32 (Database Issue): D115-D119, 2004.

34. Ru JL, Li P, Wang J, Zhou W, Li B, Huang C, Li P, Guo ZH, Tao WY, Yang YF, et al: TCMSP: A database of systems pharmacology for drug discovery from herbal medicines. J Cheminform 6: 13, 2014.

35. Xue R, Fang Z, Zhang M, Yi Z, Wen C and Shi T: TCMID: Traditional Chinese medicine inegrative database for herb molecular mechanism analysis. Nucleic Acids Res 41 (Database Issue): D1089-D1095, 2013.

36. Liu L, Liu JX, Guo H and Ren JX: Recent advances on pericytes in microvascular dysfunction and traditional Chinese medicine prevention. Zhongguo Zhong Yao Za Zhi 42: 3072-3077, 2017 (In Chinese).

37. Wang T, Jia C, Chen Y, Li X and Cheng J: Analysis on establishment and affecting factors of qi stagnation and blood stasis rat model. Zhongguo Zhong Yao Za Zhi 37: 1629-1633, 2012 (In Chinese).

38. Chen CS, Lin LW, Hsieh CC, Chen GW, Peng WH and Hsieh MT: Differential gene expression in hemodialysis patients with 'cold' zheng. Am J Chin Med 34: 377-385, 2006.

39. Lu YY, Zhao Y, Song YN, Dong S, Wei B, Chen QL, Hu YY and Su SB: Serum cytokine profiling analysis for zheng differentiation in chronic hepatitis B. Chin Med 10: 24, 2015.

40. Kiyama R: DNA microarray-based screening and characterization of traditional chinese medicine. Microarrays (Basel) 6: 4, 2017.

41. $\mathrm{Hu} X Q$ and $\mathrm{Su}$ SB: An overview of epigenetics in Chinese medicine researches. Chin J Integr Med 23: 714-720, 2017.

42. Li W, Liu P, Duan J, Ma CH, Shi XQ and Guo JM: Effects of Xiangfu Siwu decoction on nerve endocrine immune system in female rats with Qi Stagnation and Blood Stasis Syndrome. Chin J Exp Trad Med Formulae 20: 99-104, 2014.

43. Yin CE, Zhang NN, Lv FF, Guan L and Wei XJ: Study on the correlation of Qi Stagnation and Blood Stasis Syndrome and heart rate variability in patients with Coronary Heart Disease. J Sichuan Traditional Chin Med 21: 15-16, 2003 (In Chinese).
44. Ren JX, Liu JX and Lin CR: Comparative analysis on the biological basis of blood stasis syndrome induced by Qi Stagnation and Qi deficiency in patients with unstable angina pectoris. Zhongguo Zhong Xi Yi Jie He Za Zhi 30: 352-356, 2010 (In Chinese).

45. Liu JW: A preliminary bionetwork research of Qi deficiency and Qi stagnation in the context of coronary heart disease angina pectoris. Beijing Univ Chin Med, 2: 29-34, 2015.

46. Kolesnick RN: Sphingomyelin and derivatives as cellular signals. Prog Lipid Res 30: 1-38, 1991.

47. Hannun YA: The sphingomyelin cycle and the second messenger function of ceramide. J Biol Chem 269: 3125-3128, 1994.

48. Liu J, Beckman BS and Foroozesh M: A review of ceramide analogs as potential anticancer agents. Future Med Chem 5: 1405-1421, 2013.

49. Brunkhorst R, Vutukuri R and Pfeilschifter W: Fingolimod for the treatment of neurological diseases-state of play and future perspectives. Front Cell Neurosci 8: 283, 2014.

50. Arevalo JC and Wu SH: Neurotrophin signaling: Many exciting surprises. Cell Mol Life Sci 63: 1523-1537, 2006.

51. Grant BD and Donaldson JG: Pathways and mechanisms of endocytic recycling. Nat Rev Mol Cell Biol 10: 597-608, 2009.

52. Steinberg GR and Kemp BE: AMPK in health and disease. Physiol Rev 89: 1025-1078, 2009.

53. Sanchez AM, Candau RB, Csibi A, Pagano AF, Raibon A and Bernardi $\mathrm{H}$ : The role of AMP-activated protein kinase in the coordination of skeletal muscle turnover and energy homeostasis. Am J Physiol Cell Physiol 303: C475-C485, 2012.

54. Wang YY and Zhang HM: The scientific explanation of Qi. J Chin Med 58: 811-813, 2017 (In Chinese). 